\title{
Utilization of copper on iron catalyst for the synthesis of biaryl systems and benzoxazines via oxidative arylation of anilide derivatives
}

\author{
Anna Székely, Ádám Sinai, Edina B. Tóth, Zoltán Novák* \\ MTA-ELTE “Lendület” Catalysis and Organic Synthesis Research Group, Eötvös Loránd University, Institute of Chemistry, Pázmány \\ Péter stny. 1/A, H-1117 Budapest, Hungary \\ Fax: +36-1-372-2592 \\ E-mail: novakz@elte.hu
}

Received: The date will be inserted once the manuscript is accepted.

\begin{abstract}
Heterogeneous copper on iron catalyst serves as an efficient alternative copper source for arylation reactions using hypervalent iodonium salts. The copper (0) catalyst affords meta-arylation of pivalanilides, while 2-ethynylanilides undergo oxidative carboarylation-ring closure with diaryliodonium salts.
\end{abstract}

Key words: biaryls, copper, coupling, heterocycles, iron

The synthesis of biaryl systems and heterocyclic compounds via aromatic $\mathrm{C}-\mathrm{H}$ bond functionalization and oxidative ring closure play important roles in organic chemistry. The biaryl motif and heterocyclic rings are often found in natural products and biologically active compounds. ${ }^{1}$ In traditional crosscoupling reactions, prefunctionalization of the aromatic coupling partner and the utilization of metallo-organic reagents are required for efficient coupling. This chemical requirement for a coupling reaction leads to the formation of significant amount of byproducts originated from reagents, solvent or additives during the synthetic steps. To overcome these drawbacks, the use of $\mathrm{C}-\mathrm{H}$ activation reactions serves as a useful alternative in which preactivation of the aromatic compounds is not necessary. ${ }^{2}$ A variety of substituted aryl groups can be regioselectively transferred through $\mathrm{C}-\mathrm{H}$ functionalization in transition-metal catalyzed oxidative couplings with the aid of hypervalent iodonium salts. ${ }^{3}$ During the last decade several methods were developed, where diaryliodonium salts were applied for regioselective substitution of aromatic (ortho ${ }^{4}$ - and meta $^{5}$ positions) and heteroaromatic compounds (C2 and $\mathrm{C} 3$ substitution of indoles via palladium- and coppercatalysis were reported). ${ }^{6}$ The choice of the catalyst often determines the site selectivity of the reactions. ${ }^{7}$

In addition to the direct functionalization of $\mathrm{C}-\mathrm{H}$ bond with iodonium salts, several methods were developed recently for the construction of heterocycles in copper-catalyzed oxidative ring closures using iodonium salts. Based on this strategy, pyrroloindolines were synthetized via an arylationcyclization cascade by MacMillan, ${ }^{8}$ the preparation of substituted oxazines was reported by Gaunt ${ }^{9}$ and an unusual 6-exo-dig cyclization of 2-ethynyl-anilides was developed in our laboratory yielding diversely substituted benzoxazines. ${ }^{10}$

The use of $\mathrm{Cu}(\mathrm{II})$-species as precatalyst for $\mathrm{C}-\mathrm{H}$ activation reactions performed under homogeneous catalytic conditions is prevalent. $^{6,7,11}$ In these transformations, the most frequently used copper sources are $\mathrm{Cu}(\mathrm{OTf})_{2}, \mathrm{CuCl}_{2}$ and $\mathrm{CuO}$. Although, the catalyst is added to the reactions as a $\mathrm{Cu}^{2+}$ salt, it is believed that $\mathrm{Cu}(\mathrm{I})$-species are responsible for the catalytic activity. ${ }^{7,12}$ In general handling of copper(I) salts are difficult, while some of the copper(II) salts are toxic, air- and moisture sensitive, or expensive; therefore their use is not always practical.

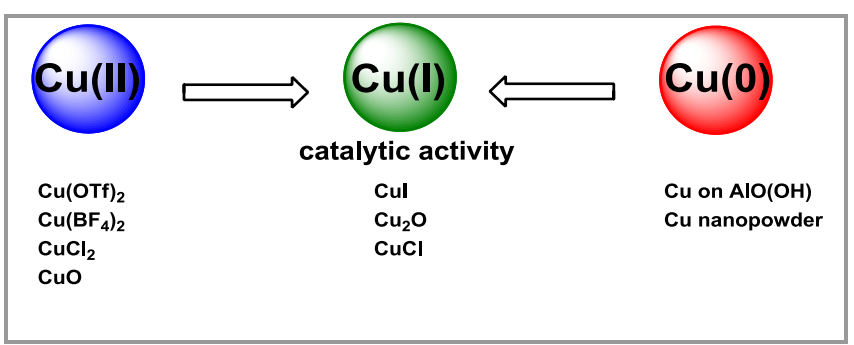

Scheme 1 Two possible approaches to the formation of $\mathrm{Cu}(\mathrm{I})$ species for $\mathrm{C}-\mathrm{H}$ activation reactions.

The catalytically active $\mathrm{Cu}(\mathrm{I})$ species can also be generated from $\mathrm{Cu}(0)$ (Scheme 1). Copper metal is cheap, easily accessible, and stable in air. Moreover, in some cases, its separation from the reaction mixture is quite simple. Interestingly, the use of elemental copper for the generation of $\mathrm{Cu}(\mathrm{I})$ species is rare in oxidative chemistry. As an example, application of $\mathrm{Cu}$ on $\mathrm{AlO}(\mathrm{OH})$ for meta-selective $\mathrm{C}-\mathrm{H}$ bond arylation was reported by Park et al. ${ }^{13}$

Previously our research group has developed a heterogeneous copper on iron catalyst, which is composed of copper nanoparticles on the surface of micron sized iron particles. The catalyst composition can be easily prepared from readily available reagents such as iron powder and $\mathrm{CuSO}_{4}$ under aqueous conditions. Our copper on iron catalyst system contains $5 \mathrm{w} / \mathrm{w} \% \mathrm{Cu}$, where the copper is electrochemically deposited on the surface of the iron. The heterogeneous catalyst can stir the reaction and also can be easily separated from the reaction mixture using an external magnetic field. Additionally, the use of $\mathrm{Cu} / \mathrm{Fe}$ catalyst significantly reduced the copper contamination of the organic products. ${ }^{14}$ The $\mathrm{Cu} / \mathrm{Fe}$ catalyst has already been successfully applied in C-S and $\mathrm{C}-\mathrm{N}$ bond forming reactions by our research group. ${ }^{15}$ 
We aimed to study the applicability of the copper on iron heterogeneous catalyst in two different novel oxidative coupling reactions utilizing hypervalent iodonium salts: 1. meta-selective arylation of pivalanilides, ${ }^{7}$ and 2 . Synthesis of benzoxazine derivatives via carboarylation-ring closure reaction of ortho ethynyl anilides (Scheme 2.). ${ }^{10}$

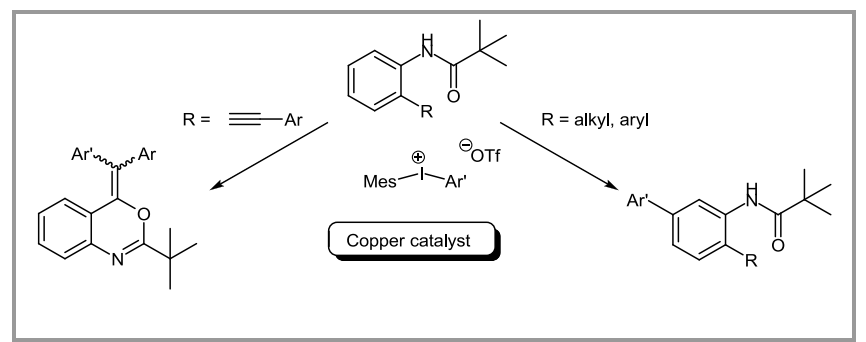

Scheme 2 Meta-selective arylation of substituted pivalanilides and oxidative ring closure of 2-arylethynyl anilides

The applicability of copper on iron catalyst for the meta-selective arylation the reaction between 2methyl-pivalanilide and phenyl(mesityl)iodonium triflates was evaluated using the reaction conditions of the homogeneous version. ${ }^{7}$ To our delight, addition of 1.5 equiv. mesityl(phenyl)iodonium triflate to the pivalanilide in the presence of $5 \mathrm{~mol} \% 5 \mathrm{w} / \mathrm{w} \% \mathrm{Cu} / \mathrm{Fe}$ provides $94 \%$ conversion (79\% isolated yield, Table 1, entry 1) in DCE in 20 hours at $50^{\circ} \mathrm{C}$. These conditions proved to be the most ideal set of parameters for the efficient coupling.

The substrate scope of the reaction was evaluated using substituted pivalanilides (1a-d) bearing different functional groups $(\mathrm{Me}, \mathrm{t}-\mathrm{Bu}, \mathrm{OMe}, \mathrm{Ph})$ in ortho position, which were prepared from commercially available anilines and pivaloyl chloride. ${ }^{16}$ The syntheses of aryl(mesityl)iodonium salts bearing COOEt, $\mathrm{F}, \mathrm{Cl}$ and $\mathrm{Br}$ groups on the aryl part (2b-e) were carried out according to the modified $^{10}$ procedure of Olofsson. ${ }^{17}$

Arylations of the four pivalanilide substrates (1a-d) were performed with substituted aryl mesityl iodonium salts (2b-e) (Table 1, Entries 2-13). Aromatic groups bearing esters function were straightforwardly introduced into the meta position of the anilides with $\mathbf{2 b}$ and the biaryl products (3b-d) were isolated in 54-83\%, depending on the substituent of the anilide. Comparing the results among the halogen-substituted aryl iodonium salts, the highest yield were obtained in case of the bromo substituted derivatives (3k-m) (70-79\%) while aromatic groups containing chloro (3h-j) and fluoro (3e-g) function gave lower yield on the average (47-74\% and 53$72 \%$ ).

After demonstrating the applicability of $\mathrm{Cu} / \mathrm{Fe}$ catalyst in meta- selective arylation, we aimed to study its catalytic activity in other oxidative coupling reaction.
Table 1 Substrate scope using different functional groups ${ }^{\mathrm{a}}$

\begin{tabular}{|c|c|c|c|c|}
\hline$\underbrace{1}_{1 a-d}$ & \multicolumn{2}{|r|}{$2 d=e$} & $\begin{array}{l}\underset{5 \mathrm{~mol} \%}{\mathrm{CU} / \mathrm{Fe}(5 \mathrm{w} / \mathrm{w} \%)} \\
\underset{50-70^{\circ} \mathrm{C}}{1,2-\mathrm{DCE}, 20-48 \mathrm{~h}}\end{array}$ & 3a-m \\
\hline Entry & $\mathbf{R}^{1}$ & $\mathbf{R}^{2}$ & Product & Yield (\%) \\
\hline 1 & $\mathrm{Me}$ & $\mathrm{H}$ & $3 a$ & 79 \\
\hline 2 & $\mathrm{Me}$ & COOEt & $\mathbf{3 b}$ & 83 \\
\hline 3 & $\mathrm{OMe}$ & COOEt & $3 c$ & 61 \\
\hline 4 & $\mathrm{Ph}$ & COOEt & 3d & 54 \\
\hline 5 & $\mathrm{Me}$ & $\mathrm{F}$ & $3 e$ & 57 \\
\hline 6 & $t-\mathrm{Bu}$ & $F$ & $3 f$ & 68 \\
\hline 7 & $\mathrm{Ph}$ & $\mathrm{F}$ & $3 g$ & 53 \\
\hline 8 & $\mathrm{Me}$ & $\mathrm{Cl}$ & $3 \mathrm{~h}$ & 65 \\
\hline 9 & $t-\mathrm{Bu}$ & $\mathrm{Cl}$ & $3 \mathbf{i}$ & 47 \\
\hline 10 & $\mathrm{Ph}$ & $\mathrm{Cl}$ & $3 \mathbf{j}$ & $74^{c}$ \\
\hline 11 & $\mathrm{Me}$ & $\mathrm{Br}$ & $3 k$ & 75 \\
\hline 12 & $t-\mathrm{Bu}$ & $\mathrm{Br}$ & 31 & 79 \\
\hline 13 & $\mathrm{Ph}$ & $\mathrm{Br}$ & $3 \mathrm{~m}$ & $70^{c}$ \\
\hline
\end{tabular}

${ }^{\mathrm{a}}$ Reaction conditions: The reactions were performed with 0.5 $\mathrm{mmol}$ pivalanilide, $0.75 \mathrm{mmol}$ aryl(mesityl)iodonium triflate and $32.2 \mathrm{mg} \mathrm{Cu} / \mathrm{Fe}$ catalyst in $2.5 \mathrm{ml} 1,2-\mathrm{DCE}$ at $50 / 70^{\circ} \mathrm{C}$ for $20-48$ hours.

${ }^{\mathrm{b}}$ yield of isolated product

carylation reaction was conducted at $70{ }^{\circ} \mathrm{C}$.

A novel procedure was developed in our laboratory for the synthesis of 4-diarylmethylene-benzoxazines through oxidative ring closure of ethynylanilides with diaryliodonium salt, where $\mathrm{Cu}(\mathrm{OTf})_{2}$ was used as catalyst (Scheme 3). In continuation of our work, we aimed to examine the applicability of $\mathrm{Cu} / \mathrm{Fe}$ catalyst in the oxidative carboarylation and ring closure reaction for the synthesis of benzoxazine derivatives.

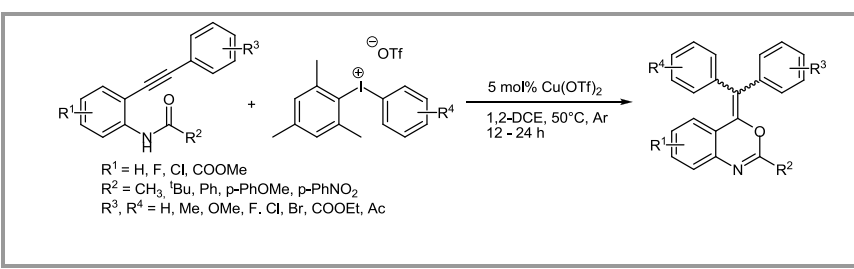

Scheme 3. Oxidative ring closure of 2-arylethynyl anilides under homogeneous catalytic conditions

The optimization reactions were carried out with $\mathrm{N}$ (2-(phenylethynyl)phenyl)pivalamide and 1.2 equiv. mesityl(phenyl)iodonium triflate varying the solvent (Table 2, entries 1-4), the catalyst loading (Table 2, entries 5-7) and the temperature (Table 2, entries 810). The highest conversion was reached when the reaction was performed in 1,2-dichloroethane at $50^{\circ} \mathrm{C}$ in the presence of $5 \mathrm{~mol} \% \mathrm{Cu} / \mathrm{Fe}$ catalyst. This finding 
is identical with the best condition applied in the homogeneous version of this transformation. ${ }^{10}$

Table 2 Optimization of reaction conditions ${ }^{2}$

\begin{tabular}{|c|c|c|c|c|}
\hline Entry & Solvent & $\mathbf{C u} / \mathbf{F e}(\mathbf{m o l} \%)$ & $\boldsymbol{T} /{ }^{\circ} \mathbf{C}$ & Conversion (\%) \\
\hline 1 & $\mathbf{T H F}$ & 5 & 50 & 6 \\
\hline 2 & $\mathbf{D M F}$ & 5 & 50 & 0 \\
\hline 3 & toluene & 5 & 50 & 45 \\
\hline 4 & $\mathbf{1 , 2 - D C E}$ & 5 & 50 & 89 \\
\hline 5 & $1,2-\mathrm{DCE}$ & $\mathbf{2 . 5}$ & 50 & 81 \\
\hline 6 & $1,2-\mathrm{DCE}$ & $\mathbf{5}$ & 50 & 89 \\
\hline 7 & $1,2-\mathrm{DCE}$ & $\mathbf{1 0}$ & 50 & 87 \\
\hline 8 & $1,2-\mathrm{DCE}$ & 5 & $\mathbf{2 5}$ & 14 \\
\hline 9 & $1,2-\mathrm{DCE}$ & 5 & $\mathbf{5 0}$ & 89 \\
\hline 10 & $1,2-\mathrm{DCE}$ & 5 & $\mathbf{8 0}$ & 76 \\
\hline
\end{tabular}

${ }^{\mathrm{a}}$ reactions were performed in $0.1 \mathrm{mmol}$ scale and GC-MS conversions were measured after 12 hours stirring under inert atmosphere.

After the optimization process, we studied the substrate scope of the procedure. Several substituted arylethynyl-anilides were treated with mesityl(phenyl)iodonium triflate in the presence of $\mathrm{Cu} / \mathrm{Fe}$ catalyst under the optimized reaction conditions. The 4-diphenylmethylenebenzoxazine (5a) was isolated with $58 \%$ yield (Table 3 , entry 1 ). The presence of electron donating methyl group in all positions on the phenyl ring in 2-arylethynyl pivalanilides resulted in increased yields compared to the unsubstituted derivative, affording the desired products 5b-d in $67 \%, 66 \%$ and $71 \%$ yield respectively (Table 3, entries 2-4). The strongly electron donating methoxy group decreased the efficiency of the reaction and compound $5 \mathbf{e}$ was isolated in $37 \%$ (Table 3, entry 5). Arylethynyl anilide containing the chlorophenyl group (Table 3, entry 6) gave product $\mathbf{5 f}(68 \%)$, while the reaction using the substrate containing the strongly electron withdrawing nitro group provided the desired product (5g) in $50 \%$ yield (Table 3, entry 7). In cases where nonsymmetrically substituted alkene derivatives are formed, the ${ }^{1} \mathrm{H}$ NMR measurements showed the presence of mixture of geometric isomers (The isomeric ratios are indicated in Table 3 ).

After functionalization of different arylethynyl anilides with phenylmesityliodonium triflate, the diversity of the product structure was expanded with the use of substituted aryl(mesityl)iodonium triflates. Using para- and meta methyl substituted iodonium salts, the appropriate products (5b and 5c) were obtained in $44 \%$ and $61 \%$ yields respectively (Table 3, entry 8,9$)$.
Table 3 Substrate scope using different functional groups.

\begin{tabular}{|c|c|c|c|c|c|c|}
\hline${ }_{3 a-g}{ }^{A}$ & & & & $\begin{array}{l}\mathrm{mol} \% \\
\mathrm{Cu} / \mathrm{Fe}(5 \mathrm{w} / \mathrm{w} \%) \\
2-\mathrm{DCE}, 50^{\circ} \mathrm{C}, \mathrm{Ar} \\
-24 \mathrm{~h}\end{array}$ & & $\frac{11}{11}$ \\
\hline Entry & $\mathbf{R}^{1}$ & $\mathbf{R}^{2}$ & $\mathbf{R}^{3}$ & Product & $\begin{array}{c}\mathrm{E} / \mathrm{Z} \\
\text { ratio }\end{array}$ & $\begin{array}{c}\text { Yield } \\
(\%)\end{array}$ \\
\hline 1 & ${ }^{\mathrm{t}} \mathrm{Bu}$ & $\mathrm{H}$ & $\mathrm{H}$ & $5 a$ & - & 58 \\
\hline 2 & ${ }^{\mathrm{t}} \mathrm{Bu}$ & $p$-Me & $\mathrm{H}$ & $5 b$ & $2 / 3$ & 67 \\
\hline 3 & ${ }^{\mathrm{t}} \mathrm{Bu}$ & $m-\mathrm{Me}$ & $\mathrm{H}$ & $5 c$ & $2 / 3$ & 66 \\
\hline 4 & ${ }^{\mathrm{t}} \mathrm{Bu}$ & $o-\mathrm{Me}$ & $\mathrm{H}$ & $5 d$ & $1 / 1.2$ & 71 \\
\hline 5 & ${ }^{\mathrm{t}} \mathrm{Bu}$ & $p$-OMe & $\mathrm{H}$ & $5 e$ & $1 / 2$ & 37 \\
\hline 6 & ${ }^{t} \mathrm{Bu}$ & $p-\mathrm{Cl}$ & $\mathrm{H}$ & $5 f$ & $1 / 2.3$ & 68 \\
\hline 7 & ${ }^{t} \mathrm{Bu}$ & $p-\mathrm{NO}_{2}$ & $\mathrm{H}$ & $5 g$ & $2 / 7$ & 50 \\
\hline 8 & ${ }^{t} \mathrm{Bu}$ & $\mathrm{H}$ & $p-\mathrm{Me}$ & $5 b$ & $3 / 2$ & 44 \\
\hline 9 & ${ }^{t} \mathrm{Bu}$ & $\mathrm{H}$ & $m$-Me & $5 c$ & $1.8 / 1$ & 61 \\
\hline 10 & ${ }^{\mathrm{t}} \mathrm{Bu}$ & $\mathrm{H}$ & $p-\mathrm{Cl}$ & $5 f$ & $9 / 5$ & 54 \\
\hline 11 & ${ }^{t} \mathrm{Bu}$ & $\mathrm{H}$ & $p-\mathrm{NO}_{2}$ & $5 \mathrm{~h}$ & - & 0 \\
\hline 12 & ${ }^{t} \mathrm{Bu}$ & $p$-Me & $p$-Me & $5 i$ & - & 31 \\
\hline 13 & ${ }^{\mathrm{t}} \mathrm{Bu}$ & $m-\mathrm{Me}$ & $m$-Me & $5 j$ & - & 61 \\
\hline 14 & ${ }^{t} \mathrm{Bu}$ & $p-\mathrm{Cl}$ & $p-\mathrm{Cl}$ & $5 k$ & - & 59 \\
\hline 15 & $\mathrm{Ph}$ & $\mathrm{H}$ & $\mathrm{H}$ & 51 & - & 70 \\
\hline
\end{tabular}

${ }^{\mathrm{a}}$ Reaction conditions: The reactions were performed with 0.35 mmol 2-arylethynyl-anilide, $0.42 \mathrm{mmol}$ aryl(mesityl)iodonium triflate and $22.2 \mathrm{mg} \mathrm{Cu} / \mathrm{Fe}$ catalyst in $3 \mathrm{ml} 1,2-\mathrm{DCE}$ at $50^{\circ} \mathrm{C}$ for 12-24 hours.

The para-chloro substituted derivative (5f) was also successfully prepared in 54\% isolated yield (Table 3, entry 10). When the reaction was carried out with (4nitrophenyl)(mesityl)iodonium triflate, the reaction did not afford the desired product (5h) (Table 3, entry 11). Compounds containing substituents on the phenyl ring of both the iodonium salt and the arylethynylanilide were also successfully reacted in the $\mathrm{Cu} / \mathrm{Fe}$ catalyzed reaction, and the expected products (5i-k) were isolated in 31-61\% yield (Table 3 , entries 12-14).

When the tert-butyl group of the amide moiety was replaced with phenyl group the reaction provided the appropriate benzoxazine product (5l) in $70 \%$ yield (Table 3, entry 15).

In conclusion, we successfully extended the application of copper on iron catalyst in the field of coupling chemistry. We have shown that $\mathrm{Cu} / \mathrm{Fe}$ can be efficiently utilized in meta-selective arylation reactions of ortho-substituted pivalanilides with diaryliodonium triflates. We also demonstrated that our copper on iron catalyst also serves as an efficient catalyst in ring closing reaction of 2 -arylethynyl anilides and hypervalent iodonium salts for the preparation of diaryl substituted methylenebenzoxazines in good yields. Furthermore the catalyst can be easily prepared, is stable in air, and is not 
sensitive to heat. Its separation from reaction mixture can be simply achieved using an external magnetic field. These properties of the catalyst would make its use advantageous in the oxidative transformations examined compared to the previously used homogenous copper sources.

${ }^{1} \mathrm{H}-\mathrm{NMR}$ and ${ }^{13} \mathrm{C}-\mathrm{NMR}$ spectra were recorded on a Bruker Avance-250 spectrometer operating at 250 $\mathrm{MHz}$ and $62.5 \mathrm{MHz}$ using DMSO- $d_{6}$ or $\mathrm{CDCl}_{3}$ as solvent. Chemical shifts are given in ppm relative to TMS, or to the residual solvent peak of DMSO and $\mathrm{CDCl}_{3}$ as internal standards. Coupling constants $(J)$ are reported in Hertz $(\mathrm{Hz})$. Infrared spectra were recorded on Bruker Alpha single-reflection diamond ATR spectrometer as solids or thin films. In the IR spectra, only the strongest/structurally most important peaks $\left(\mathrm{n}, \mathrm{cm}^{-1}\right)$ are listed. HRMS were measured on an Agilent Technologies 6210 Time of Flight mass spectrometer. Melting points were recorded on Büchi 501 apparatus and are reported uncorrected. All solvents used were distilled using standard methods. 1,2-dichloroethane were distilled from calcium hydride. All mixed solvent systems are reported as v/v solutions. All reactions were monitored by TLC using Merck DC pre coated TLC plates with $0.25 \mathrm{~mm}$ Kieselgel $60 \mathrm{~F}_{254}$. Visualization was performed with a $254 \mathrm{~nm}$ UV lamp. M-CPBA was dried under high vacuum at room temperature and was stored under argon. All other chemicals were used as received without further purification.

Supporting Information for this article is available online at http://www.thiemeconnect.com/ejournals/toc/synthesis.

\section{General procedure for the preparation of copper on iron catalyst}

A round-bottom flask was charged with iron powder (5.00 g, $89.5 \mathrm{mmol}$ ) and $\mathrm{H}_{2} \mathrm{O}$ (deoxygenated with argon) $(50 \mathrm{~mL})$. The mixture was stirred vigorously with a mechanical stirrer and a solution of $\mathrm{CuSO}_{4}$ (126 mg, $0.79 \mathrm{mmol})$ in $\mathrm{H}_{2} \mathrm{O}(50 \mathrm{~mL})$ was added dropwise under argon atmosphere over $1 \mathrm{~h}$, and the stirring was continued for $3 \mathrm{~h}$. The catalyst was separated with a magnet and washed with deoxygenated $\mathrm{H}_{2} \mathrm{O}(5 \times 20 \mathrm{~mL})$ then acetone $(3 \times 20$ $\mathrm{mL})$ and dried under reduced pressure.

\section{General procedure for the meta-selective arylation of pivalanilides:}

$0.5 \mathrm{mmol}$ pivalanilide, $0.75 \mathrm{mmol}$ iodonium salt and $32.2 \mathrm{mg} \mathrm{Cu} / \mathrm{Fe}(5 \mathrm{~mol} \%)$ was measured into a $4 \mathrm{ml}$ vial. $2.5 \mathrm{ml}$ abs. DCE was added and the reaction mixture was stirred at $50 / 70{ }^{\circ} \mathrm{C}$ for $20 / 48$ hours. Reaction mixture was separated from the catalyst with a magnetic bar, then evaporated. Purification of the crude product was achieved with column chromatography using gradient elution. If needed, further purification was done by preparative thin layer chromatography. The products were dried in vacuum oven (at $30^{\circ} \mathrm{C}$ ).

\section{N-(4-methylbiphenyl-3-yl)pivalamide (3a) ${ }^{7}$}

Followed the general procedure the reaction was performed with $57.3 \mathrm{mg}(0.3 \mathrm{mmol}) \mathrm{N}$-o-tolyl pivalanilide, $212 \mathrm{mg}(0.45 \mathrm{mmol})$ mesityl(phenyl) iodonium triflate and $19.2 \mathrm{mg}(5 \mathrm{~mol} \%) \mathrm{Cu} / \mathrm{Fe}$ for 20 $\mathrm{h}$ at $50^{\circ} \mathrm{C}$. After evaporation of the solvent the crude product was purified with column chromatography on silica gel with gradient elution (Hexanes-Ethyl acetate eluent: $75: 1 \rightarrow 50: 1 \rightarrow 25: 1 \rightarrow 20: 1 \rightarrow 10: 1 \rightarrow 5: 1)$ affording the product as white solid (63 $\mathrm{mg}, 0.24$ mmol, 79\%).

Mp $109-110{ }^{\circ} \mathrm{C}$ (lit. $110-112{ }^{\circ} \mathrm{C}$ ). $R_{f}=0.31$ (HexaneEtOAc, 8:1). )

IR (solid): 3311; 3031; 2963; 2923; 2868; 1649; 1507; $1486 ; 1455 ; 1395 ; 1275 ; 1204 ; 1165 ; 882 \mathrm{~cm}^{-1}$.

${ }^{1} \mathrm{H} \mathrm{NMR}\left(250 \mathrm{MHz}, \mathrm{CDCl}_{3}\right): \delta=1.28(\mathrm{~s}, 9 \mathrm{H}), 2.21(\mathrm{~s}$, $3 \mathrm{H}), 7.52$ (dt, $6 \mathrm{H}, J=9.4 \mathrm{~Hz}), 7.54(\mathrm{~d}, 2 \mathrm{H}, J=7.5$ $\mathrm{Hz}), 8.12$ (s, 1H).

${ }^{13} \mathrm{C} \mathrm{NMR}\left(250 \mathrm{MHz}, \mathrm{CDCl}_{3}\right): \delta=17.7 ; 28.1 ; 40.2$; $121.8 ; 123.8 ; 127.5 ; 127.6 ; 127.9 ; 129.0 ; 131.1$; $136.6 ; 140.4 ; 141.0 .177 .0$

MS (EI, $70 \mathrm{eV}): m / z(\%): 267(30 \%) ; 210(7 \%) ; 183$ (32\%); 165 (9\%); 115 (5\%); 85 (5\%); 57 (100\%).

\section{Ethyl 4'-methyl-3'-pivalamidobiphenyl-4- carboxylate $(3 b)^{7}$}

Followed the general procedure the reaction was performed with $95.6 \mathrm{mg}(0.5 \mathrm{mmol}) \mathrm{N}$-o-tolyl pivalanilide, $408 \mathrm{mg}(0.75 \mathrm{mmol})$ mesityl(4ethoxycarbonylphenyl)iodonium triflate and $32.2 \mathrm{mg}$ $(5 \mathrm{~mol} \%) \mathrm{Cu} / \mathrm{Fe}$ for $20 \mathrm{~h}$ at $50^{\circ} \mathrm{C}$. After evaporation of the solvent the remained residue was dissolved in 10 $\mathrm{ml}$ ethyl-acetate and extracted by $3 \times 10 \mathrm{ml} 1 \mathrm{M} \mathrm{NaOH}$. The aqueous phase was washed by $10 \mathrm{ml}$ ethyl-acetate and the organic phase was extracted again with $3 \times 10$ $\mathrm{ml} 1 \mathrm{M} \mathrm{NaOH}$. The two organic phases were united and dried on $\mathrm{MgSO}_{4}$. After evaporation of the solvent the crude product was purified with column chromatography on silica gel with gradient elution (Hexanes-Ethyl acetate eluent: 50:1 $\rightarrow$ 25:1 $\rightarrow$ 20:1 $\rightarrow 15: 1 \rightarrow 10: 1 \rightarrow 5: 1)$ affording the product as white solid (141 mg, $0.42 \mathrm{mmol}, 83 \%$ ).

Mp $115^{\circ} \mathrm{C} . R_{f}=0.18$ (Hexane-EtOAc, 8:1)

IR (solid): 3306; 2956; 2922; 2867; 1738; 1650; 1480; $1395 ; 1363 ; 1275 ; 1239 ; 1214 ; 1168 ; 1093 ; 1048$; $1011 ; 941 ; 907 ; 883 ; 841 \mathrm{~cm}^{-1}$.

${ }^{1} \mathrm{H}$ NMR $\left(250 \mathrm{MHz}, \mathrm{CDCl}_{3}\right): 1.36(\mathrm{~s}, 9 \mathrm{H}), 1.41(\mathrm{t}, 3 \mathrm{H}$, $\left.J^{1}=J^{2}=7.00 \mathrm{~Hz}\right), 2.30(\mathrm{~s}, 3 \mathrm{H}), 4.39\left(\mathrm{q}, 2 \mathrm{H}, J^{1}=J^{4}=\right.$ $\left.10.50 \mathrm{~Hz}, J^{2}=J^{3}=3.50 \mathrm{~Hz}\right), 7.27(\mathrm{~m}, 3 \mathrm{H}), 7.67(\mathrm{~d}, 2 \mathrm{H}$, $J=4.00 \mathrm{~Hz}), 8.07(\mathrm{~d}, 2 \mathrm{H}, J=4.25 \mathrm{~Hz}), 8.24(\mathrm{~s}, 1 \mathrm{H})$.

${ }^{13} \mathrm{C} \mathrm{NMR}\left(250 \mathrm{MHz}, \mathrm{CDCl}_{3}\right): \delta=14.8 ; 17.8 ; 28.1$; $40.6 ; 61.4 ; 121.8 ; 123.9 ; 127.2 ; 128.7 ; 129.4 ; 130.5$; $131.4 ; 136.8 ; 139.1 ; 145.5 ; 167.0 ; 177.1$. 
MS (EI, $70 \mathrm{eV}): \mathrm{m} / \mathrm{z}(\%): 339.2$ (100\%); $294.1(20 \%)$; 255.2 (51\%); 226.1 (22\%); 181.1 (22\%); 152.1 (11\%); $85.1(11 \%)$.

\section{Ethyl 4'-methoxy-3'-pivalamidobiphenyl-4- carboxylate (3c)}

Followed the general procedure the reaction was performed with $104 \mathrm{mg} \quad(0.5 \mathrm{mmol}) \quad \mathrm{N}-o-$ methoxyphenyl pivalanilide, $408 \mathrm{mg}(0.75 \mathrm{mmol})$ mesityl(4-ethoxycarbonylphenyl) iodonium triflate and $32 \mathrm{mg}(5 \mathrm{~mol} \%) \mathrm{Cu} / \mathrm{Fe}$ for $20 \mathrm{~h}$ at $50^{\circ} \mathrm{C}$. After evaporation of the solvent the remained residue was dissolved in $10 \mathrm{ml}$ ethyl-acetate and extracted with $3 \times 10 \mathrm{ml} 1 \mathrm{M} \mathrm{NaOH}$. The aqueous phase was washed with $10 \mathrm{ml}$ ethyl-acetate and the organic phase was extracted again with $3 \times 10 \mathrm{ml} 1 \mathrm{M}$. The two organic phases were united and dried on $\mathrm{MgSO}_{4}$. After evaporation of the solvent the crude product was purified with column chromatography on silica gel with gradient elution (Hexanes-Ethyl acetate eluent: $50: 1 \rightarrow 25: 1 \rightarrow 20: 1 \rightarrow 15: 1 \rightarrow 10: 1 \rightarrow 5: 1)$ affording the product as white solid (109 $\mathrm{mg}, 0.31$ $\mathrm{mmol}, 61 \%)$.

Mp $80^{\circ} \mathrm{C} . R_{f}=0.27$ (Hexane-EtOAc, $8 / 1$ ).

IR (solid): 3440; 2975; 2911; 2871; 2839; 1713; 1670; $1605 ; 1594 ; 1537 ; 1506 ; 1477 ; 1431 ; 1404 ; 1365$; $1326 ; 1286 ; 1256 ; 1175 ; 1158 ; 1127 ; 1104 ; 1051$; $1019 ; 922 ; 893 ; 859 ; 811 \mathrm{~cm}^{-1}$.

${ }^{1} \mathrm{H}$ NMR $\left(250 \mathrm{MHz}, \mathrm{CDCl}_{3}\right) \delta=1.27(\mathrm{~s}, 9 \mathrm{H}), 1.33(\mathrm{t}$, $\left.3 \mathrm{H}, J^{1}=J^{2}=7.00 \mathrm{~Hz}\right), 3.87(\mathrm{~s}, 3 \mathrm{H}), 4.31\left(\mathrm{q}, 2 \mathrm{H}, J^{1}=J^{4}\right.$ $\left.=10.75 \mathrm{~Hz}, J^{2}=J^{3}=3.5 \mathrm{~Hz}\right), 6.87(\mathrm{~d}, 1 \mathrm{H}, J=4.25$ $\mathrm{Hz}), 7.23(\mathrm{~m}, 1 \mathrm{H}), 7.60(\mathrm{~d}, 2 \mathrm{H}, 4.25 \mathrm{~Hz}), 7.97(\mathrm{~d}, 2 \mathrm{H}$, $J=4.25 \mathrm{~Hz}), 8.11(\mathrm{~s}, 1 \mathrm{H}), 8.75(\mathrm{~d}, 1 \mathrm{H}, J=1.0 \mathrm{~Hz})$.

${ }^{13} \mathrm{C}$ NMR $\left(250 \mathrm{MHz}, \mathrm{CDCl}_{3}\right): \delta=14.8 ; 28.0 ; 40.5$; $56.4 ; 61.3 ; 110.4 ; 118.8 ; 122.5 ; 127.1 ; 128.7 ; 129.0$; $130.3 ; 133.3 ; 145.4 ; 148.5 ; 167.1 ; 177.2$.

MS (EI, $70 \mathrm{eV}): \mathrm{m} / z(\%): 355$ (73\%); 310 (11\%); 271 (20\%); 256 (27\%); 228 (7\%); 200 (11\%); 183 (7\%); 167 (16\%); 139 (21\%); 85 (5\%); 57 (100\%).

HRMS: $m / z[\mathrm{M}+\mathrm{H}]^{+}$calcd for $\mathrm{C}_{21} \mathrm{H}_{25} \mathrm{NO}_{4}: 356.1856$; found: 356.1852 .

\section{Ethyl 4'-phenyl-3'-pivalamidobiphenyl-4- carboxylate (3d)}

Followed the general procedure the reaction was performed with $127 \mathrm{mg}(0.5 \mathrm{mmol})$ biphenyl-2pivalanilide, $408 \mathrm{mg}(0.75 \mathrm{mmol})$ mesityl(4ethoxycarbonylphenyl)iodonium triflate and $32 \mathrm{mg}(5$ $\mathrm{mol} \%) \mathrm{Cu} / \mathrm{Fe}$ for $20 \mathrm{~h}$ at $70^{\circ} \mathrm{C}$. After evaporation of the solvent the remained residue was dissolved in 10 $\mathrm{ml}$ ethyl-acetate and extracted with $3 \times 10 \mathrm{ml} 1 \mathrm{M}$ $\mathrm{NaOH}$. The aqueous phase was washed with $10 \mathrm{ml}$ ethyl-acetate and the organic phase was extracted again with $3 \times 10 \mathrm{ml} 1 \mathrm{M}$. The two organic phases were united and dried on $\mathrm{MgSO}_{4}$. After evaporation of the solvent the crude product was purified with column chromatography on silica gel with gradient elution (Hexanes-Ethyl acetate eluent: 50:1 $\rightarrow$ 25:1 $\rightarrow$ 20:1 $\rightarrow$ 15:1 $\rightarrow$ 10:1 $\rightarrow 5: 1)$ affording the product as white solid (108 mg, $0.27 \mathrm{mmol}, 54 \%$ ).

Mp 109-112 ${ }^{\circ} \mathrm{C} . R_{f}=0.15$ (Hexane-EtOAc, 8:1).

IR (solid): 3377; 3084; 3054; 3033; 2966; 2928; 2871; $1717 ; 1666 ; 1608 ; 1581 ; 1553 ; 1527 ; 1497 ; 1462$; $1443 ; 1421 ; 1394 ; 1367 ; 1310 ; 1270 ; 1211 ; 1177$; $1158 ; 1124 ; 1100 ; 1018 ; 1008 ; 930 ; 885 ; 856 ; 825 \mathrm{~cm}$ 1

${ }^{1} \mathrm{H}$ NMR $\left(250 \mathrm{MHz}, \mathrm{CDCl}_{3}\right) \delta=1.04(\mathrm{~s}, 9 \mathrm{H}), 1.34(\mathrm{t}$, $\left.3 \mathrm{H}, J^{1}=J^{2}=5.0 \mathrm{~Hz}\right), 4.32(\mathrm{q}, 2 \mathrm{H}, J=7.5 \mathrm{~Hz}), 7.24-$ $7.49(\mathrm{~m}, 8 \mathrm{H}), 7.67(\mathrm{~d}, 2 \mathrm{H}, J=7.5 \mathrm{~Hz}), 8.02(\mathrm{~d}, 2 \mathrm{H}, J$ $=7.5 \mathrm{~Hz}), 8.68(\mathrm{~s}, 1 \mathrm{H})$.

${ }^{13} \mathrm{C}$ NMR $\left(250 \mathrm{MHz}, \mathrm{CDCl}_{3}\right) \delta=14.7 ; 27.7 ; 40.3$; $61.3 ; 120.1 ; 123.0 ; 127.5 ; 128.7 ; 129.6 ; 130.4 ; 130.8$; 132.2; 136.1; 138.0; 140.6; 145.3; 167.0; 177.0.

MS (EI, $70 \mathrm{eV}): \mathrm{m} / \mathrm{z}(\%): 401$ (50\%); 344 (10\%); 317 (28\%); 288 (13\%); 241 (14\%); 207 (4\%); $167(5 \%)$; 85 (4\%); 57 (100\%).

HRMS: $m / z[\mathrm{M}+\mathrm{H}]^{+}$calcd for $\mathrm{C}_{26} \mathrm{H}_{27} \mathrm{NO}_{3}: 402.2064$; found: 402.2060 .

\section{N-(4'-fluoro-4-methylbiphenyl-3-yl)pivalamide $(3 \mathrm{e})^{19}$}

Followed the general procedure the reaction was performed with $95.6 \mathrm{mg}(0.5 \mathrm{mmol}) \mathrm{N}-o$-tolyl pivalanilide, $368 \mathrm{mg} \quad(0.75 \mathrm{mmol})$ mesityl(4fluorophenyl) iodonium triflate and $32.2 \mathrm{mg}(5 \mathrm{~mol} \%)$ at $50{ }^{\circ} \mathrm{C}$ for $20 \mathrm{~h}$. After evaporation of the solvent the crude product was purified with column chromatography on silica gel with gradient elution (Toluene-Hexanes-Ethyl acetate eluent : 25:25:1 $\rightarrow$ 12.5:12.5:1 $\rightarrow$ 5:5:1) affording the product as yellow oil (purity 78\%). For further purification the oil was dissolved in ethyl acetate and it was purified by preparative thin layer chromatography using Toluene: $\mathrm{MeOH}=10 / 1$ as eluent. The product was obtained as white solid ( $81 \mathrm{mg}, 0.28 \mathrm{mmol}, 57 \%)$.

Mp 107-109 ${ }^{\circ} \mathrm{C} . R_{f}=0.61$ (Toluene-MeOH, 10:1).

IR (solid): 3299; 3034; 2962; 2928; 2870; 1649; 1598; $1571 ; 1510 ; 1492 ; 1455 ; 1389 ; 1367 ; 1289 ; 1262$; $1222 ; 1202 ; 1158 ; 1096 ; 1014 ; 932 ; 876 ; 839 \mathrm{~cm}^{-1}$.

${ }^{1} \mathrm{H}$ NMR $\left(250 \mathrm{MHz}, \mathrm{CDCl}_{3}\right): \delta=1.27(\mathrm{~s}, 9 \mathrm{H}), 2.19(\mathrm{~s}$, $3 \mathrm{H}), 6.99\left(\mathrm{t}, 2 \mathrm{H}, J^{1}=J^{2}=8.5 \mathrm{~Hz}\right), 7.14(\mathrm{~s}, 2 \mathrm{H}), 7.26$ (s, 1H), $7.47(\mathrm{~m}, 2 \mathrm{H}), 8.07(\mathrm{~s}, 1 \mathrm{H})$.

${ }^{13} \mathrm{C} \mathrm{NMR}\left(250 \mathrm{MHz}, \mathrm{CDCl}_{3}\right): \delta=17.7 ; 28.1 ; 40.2$; $121.8 ; 123.8 ; 127.5 ; 127.9 ; 129.0 ; 131.1 ; 136.6$; $140.4 ; 141.0 ; 177.1$.

MS (EI, $70 \mathrm{eV}): \mathrm{m} / \mathrm{z}(\%): 285.1$ (54.2\%); 228.1 (4.2\%); 201.1 (45.8\%); 170.1 (7.5\%); 133.1 (4.2\%); 85.1 (6.7\%); $57.1(100 \%)$.

\section{N-(4-tert-butyl-4'-fluorobiphenyl-3-yl)pivalamide (3f)}

Followed the general procedure the reaction was performed with $116.6 \mathrm{mg}(0.5 \mathrm{mmol}) \quad \mathrm{N}-o-t-$ butylphenyl pivalanilide, $368 \mathrm{mg}(0.75 \mathrm{mmol})$ mesityl(4-fluorophenyl) iodonium triflate and $32.2 \mathrm{mg}$ 
$(5 \mathrm{~mol} \%) \mathrm{Cu} / \mathrm{Fe}$ at $50^{\circ} \mathrm{C}$ for $20 \mathrm{~h}$. After evaporation of the solvent the crude product was purified with column chromatography on silica gel (Toluene: Hexanes-Ethyl-acetate eluent in 2.5:12.5:1 ratio) affording the product as yellow oil (purity: $82 \%$ ). For further purification the oil was dissolved in ethyl acetate and it was purified by preparative thin layer chromatography using Toluene-MeOH, 10:1 as eluent. The product was obtained as white solid (111 mg, 0.34 mmol, 68\%).

Mp $142^{\circ} \mathrm{C} . R_{f}=0.65$ (Toluene:MeOH, 10:1).

IR (solid): 3265; 3004; 2960; 2932; 2870; 1645; 1507; $1492 ; 1476 ; 1418 ; 1398 ; 1367 ; 1280 ; 1218 ; 1173$; $1158 ; 1095 ; 1046 ; 1027 ; 1014 ; 943 ; 909 ; 882 ; 842$; $820 \mathrm{~cm}^{-1}$;.

${ }^{1} \mathrm{H}$ NMR $\left(250 \mathrm{MHz}, \mathrm{CDCl}_{3}\right): \delta=1.29(\mathrm{~s}, 9 \mathrm{H}), 1.37(\mathrm{~s}$, $9 \mathrm{H}), 7.00\left(\mathrm{t}, 2 \mathrm{H}, J^{1}=J^{2}=8.75 \mathrm{~Hz}\right), 7.20(\mathrm{~m}, 1 \mathrm{H})$, $7.36(\mathrm{~d}, 1 \mathrm{H}, J=4.25 \mathrm{~Hz}), 7.47(\mathrm{~m}, 3 \mathrm{H}), 7.88(\mathrm{~d}, 1 \mathrm{H}, J$ $=2.0 \mathrm{~Hz}$ ).

${ }^{13} \mathrm{C}$ NMR $\left(250 \mathrm{MHz}, \mathrm{CDCl}_{3}\right): \delta=28.1 ; 31.0 ; 34.7$; $40.0 ; 115.7 ; 116.1 ; 124.2 ; 125.9 ; 127.4 ; 129.0 ; 129.1$; $136.4 ; 139.1 ; 140.8 ; 176.7$.

MS (EI, $70 \mathrm{eV}): \mathrm{m} / z$ (\%):327 (21\%); 270 (100\%); 228 (23\%); 185 (9\%); 57 (82\%).

HRMS: $\mathrm{m} / \mathrm{z}[\mathrm{M}+\mathrm{Na}]^{+}$calcd for $\mathrm{C}_{21} \mathrm{H}_{26} \mathrm{NOF}$ : 350.1891; found: 350.1884 .

\section{N-(4'-fluoro-4-phenylbiphenyl-3-yl)pivalamide(3g)}

Followed the general procedure the reaction was performed with $127 \mathrm{mg}(0.5 \mathrm{mmol})$ biphenyl-2pivalanilide, $368 \mathrm{mg} \quad(0.75 \mathrm{mmol})$ mesityl(4fluorophenyl) iodonium triflate and $32.2 \mathrm{mg}(5 \mathrm{~mol} \%)$ $\mathrm{Cu} / \mathrm{Fe}$ at $50^{\circ} \mathrm{C}$ for $20 \mathrm{~h}$. After evaporation of the solvent the remaining residue was purified by flash chromatography on silica gel (Eluent: Hexanes-Ethyl acetate, 5:1) and the product was isolated with $71 \%$ purity as yellow oil. For further purification the oil was dissolved in ethyl acetate and it was purified by preparative thin layer chromatography using Toluene: Methanol $=10: 1$ as eluent. The pure product was obtained as white solid (92 mg, $0.26 \mathrm{mmol}, 53 \%$ ).

Mp $115^{\circ} \mathrm{C} . R_{f}=0.65$ (Hexane-EtOAc, 5:1); $\mathrm{Rf}=0.86$ (Toluene-MeOH, 10:1).

IR (solid): 3428; 3064; 2970; 2955; 2928; 2864; $1684 ; 1597 ; 1560 ; 1533 ; 1502 ; 1460 ; 1443 ; 1422$; $1394 ; 1361 ; 1306 ; 1211 ; 1155 ; 1122 ; 1096 ; 1076$; $1028 ; 1008 ; 919 ; 898 ; 846 ; 821 \mathrm{~cm}^{-1}$.

${ }^{1} \mathrm{H}$ NMR $\left(250 \mathrm{MHz}, \mathrm{CDCl}_{3}\right): \delta=1.13(\mathrm{~s}, 9 \mathrm{H}), 7.12(\mathrm{t}$, $\left.2 \mathrm{H}, J^{1}=J^{2}=7.5 \mathrm{~Hz}\right), 7.50(\mathrm{~m}, 8 \mathrm{H}), 7.66(\mathrm{t}, 2 \mathrm{H}, J=$ $7.5 \mathrm{~Hz}), 8.70(\mathrm{~s}, 1 \mathrm{H})$.

${ }^{13} \mathrm{C} \mathrm{NMR}\left(250 \mathrm{MHz}, \mathrm{CDCl}_{3}\right): \delta=28.0 ; 40.4 ; 115.8$; $116.2 ; 119.6 ; 119.7 ; 122.7 ; 128.6 ; 129.1 ; 129.3 ; 129.6$; $129.7 ; 131.4 ; 136.1 ; 138.2 ; 141.0 ; 177.0$.

MS (EI, $70 \mathrm{eV}): \mathrm{m} / z(\%): 347$ (92\%); 290 (18\%); 263 (69\%); 241 (8\%); 167 (8\%); 57 (100\%).

HRMS: $\mathrm{m} / \mathrm{z}[\mathrm{M}+\mathrm{Na}]^{+}$calcd for $\mathrm{C}_{23} \mathrm{H}_{22} \mathrm{NOF}$ : 370.1578; found: 370.1583 .

\section{N-(4'-chloro-4-methylbiphenyl-3-yl)pivalamide}

(3h)

Followed the general procedure the reaction was performed with $95.6 \mathrm{mg}(0.5 \mathrm{mmol}) \quad \mathrm{N}$-o-tolyl pivalanilide, $380 \mathrm{mg} \quad(0.75 \mathrm{mmol})$ mesityl(4chlorophenyl) iodonium triflate and $32.2(5 \mathrm{~mol} \%) \mathrm{mg}$ $\mathrm{Cu} / \mathrm{Fe}$ at $50^{\circ} \mathrm{C}$ for $20 \mathrm{~h}$. After evaporation of the solvent the crude product was purified with column chromatography on silica gel (Toluene: Hexanes: Ethyl acetate eluent in the ratio of 12.5:12.5:1) affording the product as yellow oil (purity:78\%). For further purification the yellow oil was dissolved in ethyl acetate and it was purified by preparative thin layer chromatography using Toluene: Methanol $=10: 1$ as eluent. The product was obtained as white solid ( 85 $\mathrm{mg}, 0.28 \mathrm{mmol}, 65 \%)$.

Mp $148^{\circ} \mathrm{C} . R_{f}=0.63$ (Toluene-MeOH, 10:1).

IR (solid): 3312; 3031; 2965; 2924; 2901; 2860; $1649 ; 1612 ; 1517 ; 1499 ; 1479 ; 1455 ; 1397 ; 1382$; $1364 ; 1309 ; 1285 ; 1222 ; 1202 ; 1165 ; 1130 ; 1093$; 1083; 1038; 1011; 990; 957; 930; 906; 882; 834; 809; $768 \mathrm{~cm}^{-1}$.

${ }^{1} \mathrm{H}$ NMR $\left(250 \mathrm{MHz}, \mathrm{CDCl}_{3}\right): \delta=1.27(\mathrm{~s}, 9 \mathrm{H}), 2.19(\mathrm{~s}$, $3 \mathrm{H}), 7.15$ (brs, 2H), 7.27 (d, 3H, J = 4.25 Hz), 7.44 (d, $2 \mathrm{H}, J=4.25 \mathrm{~Hz}), 8.09(\mathrm{~s}, 1 \mathrm{H})$.

${ }^{13} \mathrm{C}$ NMR $\left(250 \mathrm{MHz}, \mathrm{CDCl}_{3}\right): \delta=17.7 ; 28.1 ; 40.3$; $121.5 ; 123.5 ; 128.1 ; 128.7 ; 129.2 ; 131.3 ; 133.6$; $136.8 ; 139.0 ; 139.5 ; 177.1$.

MS (EI, $70 \mathrm{eV}): \mathrm{m} / \mathrm{z}$ (\%): 301 (27\%); 244 (4\%); 217 (19\%); 180 (7\%); 152 (8\%); 85 (7\%); 57 (100\%).

HRMS: $m / z[\mathrm{M}+\mathrm{H}]^{+}$calcd for $\mathrm{C}_{18} \mathrm{H}_{20} \mathrm{NOCl}$ : 302.1306; found: 302.1299 .

\section{N-(4-tert-butyl-4'-chlorobiphenyl-3-yl)pivalamide}

(3i)

Followed the general procedure the reaction was performed with $116.6 \mathrm{mg}(0.5 \mathrm{mmol}) \mathrm{N}$-o-tolyl pivalanilide, $380 \mathrm{mg} \quad(0.75 \mathrm{mmol})$ mesityl(4chlorophenyl) iodonium triflate and $32.2 \mathrm{mg}$ (5 mol\%) $\mathrm{Cu} / \mathrm{Fe}$ at $50^{\circ} \mathrm{C}$ for $20 \mathrm{~h}$. After evaporation of the solvent purification of the crude product with flash chromatography on silica gel (Hexanes-Ethyl acetate, $5: 1$, as eluent) afforded the product as yellow oil (Purity: 79\%). The yellow oil was dissolved in ethyl acetate and it was purified by preparative thin layer chromatography using Toluene-Methanol, 10:1 as eluent. The product is afforded as white solid $(81 \mathrm{mg}$, $0.24 \mathrm{mmol}, 47 \%$ ).

Mp $122-144^{\circ} \mathrm{C} . R_{f}=0.37$ (Hexane-EtOAc, $\left.5: 1\right) ; R_{f}=$ 0.74 (Toluene-MeOH, 10:1).

IR (solid): 3306; 2956; 2922; 2867; 1738; 1650; $1480 ; 1395 ; 1363 ; 1275 ; 1239 ; 1214 ; 1168 ; 1093$; $1048 ; 1011 ; 941 ; 907 ; 883 ; 841 \mathrm{~cm}^{-1}$.

${ }^{1} \mathrm{H}$ NMR $\left(250 \mathrm{MHz}, \mathrm{CDCl}_{3}\right): \delta=1.29(\mathrm{~s}, 9 \mathrm{H}), 1.37$ (s, 9H), $7.25(\mathrm{~m}, 3 \mathrm{H}), 7.37(\mathrm{~d}, 1 \mathrm{H}, J=4.13 \mathrm{~Hz}), 7.45(\mathrm{~d}$, $3 \mathrm{H}, J=4.25 \mathrm{~Hz}), 7.90(\mathrm{~d}, 1 \mathrm{H}, J=1.0 \mathrm{~Hz})$. 
${ }^{13} \mathrm{C}$ NMR $\left(250 \mathrm{MHz}, \mathrm{CDCl}_{3}\right): \delta=28.1 ; 31.0 ; 34.8$; $40.0 ; 124.2 ; 125.8 ; 127.5 ; 128.7 ; 129.2 ; 133.7 ; 136.5$; $138.8 ; 139.1 ; 141.2 ; 176.7$.

MS (EI, $70 \mathrm{eV}): \mathrm{m} / \mathrm{z}(\%): 343$ (15\%); $286(100 \%)$; 244 (18\%); 193 (8\%); 165 (11\%); 57 (100\%).

HRMS: $m / z[\mathrm{M}+\mathrm{Na}]^{+}$calcd for $\mathrm{C}_{21} \mathrm{H}_{26} \mathrm{NOCl}$ : 366.1595; found: 366.1593 .

\section{N-(4'-chloro-4-phenylbiphenyl-3-yl)pivalamide (3j)}

Followed the general procedure the reaction was performed with $127 \mathrm{mg}$ (0.5 mmol) biphenyl-2pivalanilide, $380 \quad \mathrm{mg} \quad(0.75 \mathrm{mmol})$ mesityl(4chlorophenyl) iodonium triflate and $32 \mathrm{mg}(5 \mathrm{~mol} \%)$ $\mathrm{Cu} / \mathrm{Fe}$ at $70^{\circ} \mathrm{C}$ for $20 \mathrm{~h}$. After evaporation of the solvent purification of the crude product with flash chromatography on silica gel (Hexanes-Ethyl acetate, 5:1 as eluent) the product as yellow oil (purity: $86 \%$ ). For further purification the yellow oil was dissolved in ethyl acetate and it was purified by preparative thin layer chromatography using Toluene-Methanol, 10:1 as eluent. The product was obtained as white solid (135 mg, $0.37 \mathrm{mmol}, 74 \%)$.

Mp $137^{\circ} \mathrm{C} . R_{f}=0.48$ (Hexane-EtOAc, $2: 1$ ). $R_{f}=0.75$ (Toluene-MeOH, 20:1).

IR (solid): 3434; 3383; 3038; 2965; 2946; 2929; $2867 ; 1677 ; 1581 ; 1551 ; 1523 ; 1486 ; 1470 ; 1460$; $1442 ; 1418 ; 1385 ; 1303 ; 1277 ; 1249 ; 1208 ; 1154$; $1127 ; 1087 ; 1025 ; 1007 ; 929 ; 882 \mathrm{~cm}^{-1}$.

${ }^{1} \mathrm{H} \mathrm{NMR}\left(250 \mathrm{MHz}, \mathrm{CDCl}_{3}\right): \delta=1.13 \mathrm{ppm}(\mathrm{s}, 9 \mathrm{H})$, $7.30-7.65 \mathrm{ppm}(\mathrm{m}, 12 \mathrm{H}), 8.71 \mathrm{ppm}(\mathrm{d}, J=1.5 \mathrm{~Hz}$, $1 \mathrm{H})$.

${ }^{13} \mathrm{C}$ NMR $\left(250 \mathrm{MHz}, \mathrm{CDCl}_{3}\right): \delta=27.5 ; 40.0 ; 119.4$; $122.3 ; 128.4 ; 128.6 ; 129.0 ; 129.3 ; 129.4 ; 130.3$; $131.4 ; 133.7 ; 135.8 ; 137.7 ; 139.1 ; 140.2 ; 176.5$.

MS (EI, $70 \mathrm{eV}): \mathrm{m} / \mathrm{z}(\%): 363$ (28\%); 306 (6\%); 279 (28\%); 243 (13\%); 85 (6\%); 57 (100\%).

HRMS: $m / z[\mathrm{M}+\mathrm{Na}]^{+}$calcd for $\mathrm{C}_{23} \mathrm{H}_{22} \mathrm{NOCl}$ : 386.1282; found: 386.1283 .

\section{N-(4'-bromo-4-methylbiphenyl-3-yl)pivalamide $(3 \mathbf{k})^{13}$}

Followed the general procedure the reaction was performed with $95.5 \mathrm{mg}(0.5 \mathrm{mmol})$ biphenyl-2pivalanilide, $413.4 \mathrm{mg}(0.75 \mathrm{mmol})$ mesityl(4bromophenyl) iodonium triflate and $32.5 \mathrm{mg}$ (5 $\mathrm{mol} \%) \mathrm{Cu} / \mathrm{Fe}$ at $50^{\circ} \mathrm{C}$ for $20 \mathrm{~h}$. After evaporation of the solvent purification of the crude product with flash chromatography on silica gel (Hexanes-Ethyl acetate, 2:1 as eluent) afforded the product as yellow oil (purity: 89\%). The yellow oil was solved in ethyl acetate and it was purified by preparative thin layer chromatography using Toluene-Methanol, 20:1 as eluent. $\mathrm{Rf}=0.62$. The product is afforded as white solid (129 mg, $0.37 \mathrm{mmol}, 75 \%$ ).

Mp $141-142^{\circ} \mathrm{C} . \quad R_{f}=0.68$ (Hexane-EtOAc, 2:1). (Toluene-MeOH, 20:1).
IR (solid): 3312; 3030; 2965; 2924; 2861; 1738; $1649 ; 1479 ; 1378 ; 1273 ; 1241 ; 1222 ; 1202 ; 1165$; 1070; 1044; $1007 ; 930 ; 905 ; 882 ; 884 \mathrm{~cm}^{-1}$.

${ }^{1} \mathrm{H}$ NMR $\left(250 \mathrm{MHz}, \mathrm{CDCl}_{3}\right): \delta=1.36(\mathrm{~s}, 9 \mathrm{H}), 2.28(\mathrm{~s}$, $3 \mathrm{H}), 7.24 \mathrm{ppm}\left(\mathrm{t}, 2 \mathrm{H}, J^{1}=J^{2}=10.0 \mathrm{~Hz}\right), 7.50(\mathrm{~m}$, $4 \mathrm{H}), 7.65 \mathrm{ppm}(\mathrm{q}, 2 \mathrm{H}, J=7.5 \mathrm{~Hz}), 8.15 \mathrm{ppm}(\mathrm{s}, 1 \mathrm{H})$.

${ }^{13} \mathrm{C}$ NMR $\left(250 \mathrm{MHz}, \mathrm{CDCl}_{3}\right): \delta=17.7 ; 28.2 ; 40.4$; $121.5 ; 121.8 ; 123.6 ; 128.4 ; 129.0 ; 131.3 ; 132.1$; $136.7 ; 139.0 ; 140.0 ; 177.2$.

MS (EI, $70 \mathrm{eV}): \mathrm{m} / z$ (\%): 345 (15\%); 261 (14\%); 180 (9\%); 153 (11\%); 85 (6\%); 57 (100\%).

\section{N-(4'-bromo-4-tert-butylbiphenyl-3-yl)pivalamide} (3l)

Followed the general procedure the reaction was performed with $116.6 \mathrm{mg}(0.5 \mathrm{mmol}) \mathrm{N}-o$-t-butylphenyl pivalanilide, $413.4 \mathrm{mg}(0.75 \mathrm{mmol})$ mesityl(4bromophenyl) iodonium triflate and $32.5 \mathrm{mg}$ (5 $\mathrm{mol} \%) \mathrm{Cu} / \mathrm{Fe}$ at $50^{\circ} \mathrm{C}$ for $20 \mathrm{~h}$. After evaporation of the solvent purification of the crude product with flash chromatography on silica gel (Hexanes-Ethyl acetate, 5:1 as eluent) afforded the product as yellow oil (purity 78\%). For further purification the yellow oil was dissolved in ethyl acetate and it was purified by preparative thin layer chromatography using TolueneMethanol, 10:1 as eluent. The product was obtained as white solid (153 mg, $0.39 \mathrm{mmol}, 79 \%$ ).

Mp $126^{\circ} \mathrm{C} . R_{f}=0.40$ (Hexane-EtOAc, 5:1). $R_{f}=0.74$ (Toluene-MeOH, 20:1)

IR (solid): 3305; 2956; 2921; 2902; 2867; 1737; $1650 ; 1477 ; 1394 ; 1361 ; 1273 ; 1238 ; 1214 ; 1167$; $1095 ; 1070 ; 1048 ; 1007 ; 941 ; 906 ; 883 \mathrm{~cm}^{-1}$.

${ }^{1} \mathrm{H}$ NMR $\left(250 \mathrm{MHz}, \mathrm{CDCl}_{3}\right) \delta=1.30(\mathrm{~s}, 9 \mathrm{H}), 1.37(\mathrm{~s}$, $9 \mathrm{H}), 7.21-7.32(\mathrm{~m}, 1 \mathrm{H}), 7.46-7.54(\mathrm{~m}, 6 \mathrm{H}), 7.91$ (d, $J=2.0 \mathrm{~Hz}, 1 \mathrm{H})$.

${ }^{13} \mathrm{C}$ NMR $\left(250 \mathrm{MHz}, \mathrm{CDCl}_{3}\right) \delta=27.8 ; 30.7 ; 34.5$; $39.7 ; 121.6 ; 123.9 ; 125.5 ; 127.3 ; 128.8 ; 131.9 ; 136.2$; $138.5 ; 139.3 ; 141.0 ; 176.5$.

MS (EI, $70 \mathrm{eV}): \mathrm{m} / \mathrm{z}(\%): 387$ (22\%); $330(100 \%)$; 288 (24\%); 251 (9\%); 209 (7\%); 193 (15\%); 165 (17\%); 139 (7\%); 57 (85\%).

HRMS: $m / z[\mathrm{M}+\mathrm{H}]^{+}$calcd for $\mathrm{C}_{21} \mathrm{H}_{26} \mathrm{NOBr}$ : 388.1271; found: 388.1263 .

\section{N-(4'-bromo-4-phenylbiphenyl-3-yl)pivalamide (3m)}

Followed the general procedure the reaction was performed with $127 \mathrm{mg}(0.5 \mathrm{mmol})$ biphenyl-2pivalanilide, $413.4 \mathrm{mg}(0.75 \mathrm{mmol})$ mesityl(4bromophenyl) iodonium triflate and $32.5 \mathrm{mg}$ (5 $\mathrm{mol} \%) \mathrm{Cu} / \mathrm{Fe}$ at $50^{\circ} \mathrm{C}$ for $20 \mathrm{~h}$. After evaporation of the solvent purification of the crude product with flash chromatography on silica gel (Hexanes-Ethyl acetate, 5:1 as eluent) afforded the product as yellow oil (purity: 77\%). For further purification the yellow oil was dissolved in ethyl acetate and it was purified by preparative thin layer chromatography using Toluene- 
Methanol, 10:1 as eluent. The product was obtained as white solid (143 mg, $0.35 \mathrm{mmol}, 70 \%$ ).

Mp $119^{\circ} \mathrm{C} . R_{f}=0.60$ (Hexane-EtOAc, 5:1). $R_{f}=0.86$ (Toluene-MeOH, 20:1).

IR (solid): 3430; 3081; 3062; 3023; 2970; 2953; 2926; $2864 ; 1743 ; 1684 ; 1578 ; 1555 ; 1530 ; 1477$; $1460 ; 1445 ; 1421 ; 1384 ; 1304 ; 1279 ; 1245 ; 1209$; $1160 ; 1141 ; 1124 ; 1069 ; 1004 ; 919 ; 896 \mathrm{~cm}^{-1}$.

${ }^{1} \mathrm{H}$ NMR $\left(250 \mathrm{MHz}, \mathrm{CDCl}_{3}\right) \delta=1.13(\mathrm{~s}, 9 \mathrm{H}), 7.12-$ $7.74(\mathrm{~m}, 12 \mathrm{H}), 8.71(\mathrm{~s}, 1 \mathrm{H})$.

${ }^{13} \mathrm{C}$ NMR $\left(250 \mathrm{MHz}, \mathrm{CDCl}_{3}\right) \delta=27.5 ; 40.0 ; 119.3$; $121.9 ; 122.3 ; 128.4 ; 128.9 ; 129.3 ; 129.4 ; 130.4$; $131.5 ; 131.9 ; 135.8 ; 137.7 ; 139.6 ; 140.2 ; 176.7$.

MS (EI, $70 \mathrm{eV}): \mathrm{m} / z$ (\%): 409 (25\%); 407 (25\%); 323 (25\%); 241 (19\%); 57 (100\%).

HRMS: $m / z[\mathrm{M}+\mathrm{Na}]^{+}$calcd for $\mathrm{C}_{23} \mathrm{H}_{22} \mathrm{NOBr}$ : 430.0777; found: 430.0780 .

General procedure for the synthesis of 2-tert-butyl4-(diarylmethylene)-4H-benzo[d][1,3] oxazines

$\mathrm{N}$-(2-(arylylethynyl)phenyl)pivalamide $(0.35 \mathrm{mmol}$, 1.0 equiv.), aryl(mesityl)iodonium trifluoromethanesulfonate $(0.42 \mathrm{mmol}, 1.2$ equiv. $)$ and $\mathrm{Cu} / \mathrm{Fe}$ (22.2 mg, $0.0175 \mathrm{mmol}, 5 \mathrm{~mol} \%)$ were placed in a $4 \mathrm{ml}$ vial, which was sealed with rubber septa, evacuated then charged with argon. 1,2dichloroethane $(3 \mathrm{ml})$ was added, and the reaction mixture was stirred at $50^{\circ} \mathrm{C}$ for the indicated time. The catalyst was separated from the reaction mixture with a magnet. The reaction mixture was diluted with $\mathrm{CH}_{2} \mathrm{Cl}_{2}(15 \mathrm{ml})$, washed twice with saturated sodium bicarbonate solution $(15 \mathrm{ml})$, the aqueous phase was extracted with $\mathrm{CH}_{2} \mathrm{Cl}_{2}(15 \mathrm{ml})$, and the combined organic phases were dried over magnesium sulphate and evaporated in vacuo. The crude product was purified by column chromatography.

\section{2-tert-butyl-4-(diphenylmethylene)-4H- benzo[d][1,3]oxazine ${ }^{10}(5 a)$}

Prepared according to the general procedure from N(2-(phenylethynyl)phenyl)pivalamide $(97.1 \mathrm{mg}, 0.35$ mmol) with mesityl(phenyl)iodonium trifluoromethanesulfonate $(198.4 \mathrm{mg}, 0.42 \mathrm{mmol})$. Reaction time: $12 \mathrm{~h}$. The crude product was purified by column chromatography on Brockmann II type neutral alumina with hexanes/ dichloromethane 100/ 1 to give a yellow solid (72 $\mathrm{mg}, 0.203 \mathrm{mmol}, 58 \%$ ).

Mp $167-169{ }^{\circ}$ C. $R_{f}: 0.72$ (hexanes-ethyl acetate, $7: 1)$;

IR (solid): 2975; 1640; 1615; 1597; 1456; 1219; 1136; $1061 \mathrm{~cm}^{-1}$.

${ }^{1} \mathrm{H}$ NMR $\left(250 \mathrm{MHz}, \mathrm{CDCl}_{3}\right): \delta=1.02 \mathrm{ppm}(\mathrm{s}, 9 \mathrm{H}$, $\left.\mathrm{CH}_{3}\right) ; 6.54 \mathrm{ppm}(\mathrm{d}, J=7.7 \mathrm{~Hz}, 1 \mathrm{H}, \mathrm{Ar}-\mathrm{H}) ; 6.68 \mathrm{ppm}$ (ddd, $J=8.6 \mathrm{~Hz}$ and $5.9 \mathrm{~Hz}$ and $2.7 \mathrm{~Hz}, 1 \mathrm{H}, \mathrm{Ar}-\mathrm{H}$ ); $7.07 \mathrm{ppm}(\mathrm{dd}, J=5.1 \mathrm{~Hz}$ and $2.0 \mathrm{~Hz}, 1 \mathrm{H}, \mathrm{Ar}-\mathrm{H}) ; 7.10$ - $7.16 \mathrm{ppm}(\mathrm{m}, 2 \mathrm{H}, \mathrm{Ar}-\mathrm{H}) ; 7.17-7.22 \mathrm{ppm}(\mathrm{m}, 8 \mathrm{H}$, Ar-H).
${ }^{13} \mathrm{C}$ NMR $\left(62.5 \mathrm{~Hz}, \mathrm{CDCl}_{3}\right): \delta=27.5 ; 37.3 ; 120.7$; $121.6 ; 125.6 ; 126.0 ; 126.8 ; 127.0 ; 127.5 ; 127.8$; $129.1 ; 129.9 ; 130.0 ; 130.9 ; 140.1 ; 140.6 ; 141.3$; $141.6 ; 167.1$.

MS (EI, $70 \mathrm{eV}): \mathrm{m} / \mathrm{z}(\%): 353(100 \%) ; 296(45 \%)$; 280 (10\%); 268 (27\%); 165 (36\%); 57 (9\%).

\section{(E/Z)-4-((4-methylphenyl)(phenyl)methylene)-2- tert-butyl-4H-benzo[d][1,3]oxazine ${ }^{10}(5 \mathrm{~b})$}

Prepared according to the general procedure from $\mathrm{N}$ (2-((4-methylphenyl)ethynyl)phenyl)pivalamide (102.0 mg, $0.35 \mathrm{mmol}$ ) with mesityl(phenyl)iodonium trifluoromethanesulfonate $(198.4 \mathrm{mg} 0.42 \mathrm{mmol})$. Reaction time: $12 \mathrm{~h}$. The crude product was purified by column chromatography on Brockmann II type neutral alumina with hexanes-dichloromethane, 100:1 to give a pale yellow solid ( $87 \mathrm{mg}, 0.24 \mathrm{mmol}, 67 \%)$. Isomer ratio $\mathrm{E} / \mathrm{Z}=2 / 3$ (from ${ }^{1} \mathrm{H} \mathrm{NMR}$ ).

Prepared according to the general procedure from N(2-(phenylethynyl)phenyl)pivalamide (97.1 $\mathrm{mg}, 0.35$ mmol) with 4-methylphenyl(mesityl)iodonium trifluoromethanesulfonate (204.2 $\mathrm{mg} 0.42 \mathrm{mmol})$, Reaction time: $18 \mathrm{~h}$. The crude product was purified by column chromatography on Brockmann II type neutral alumina with hexanes/ dichloromethane 100/ 1 to give a pale yellow solid (57 $\mathrm{mg}, 0.16 \mathrm{mmol}, 44 \%)$. Isomer ratio $\mathrm{E} / \mathrm{Z}=3 / 2$ (from ${ }^{1} \mathrm{H} \mathrm{NMR}$ ).

Mp $108-110{ }^{\circ} \mathrm{C} ; R_{f}: 0.73$ (hexanes-ethyl acetate, $7: 1)$;

IR (solid): 2959, 2925, 1636, 1594, 1455, 1275, 1219 , $1140,1059,828,761,695 \mathrm{~cm}^{-1}$.

${ }^{1} \mathrm{H}$ NMR $\left(250 \mathrm{MHz}, \mathrm{CDCl}_{3}\right): \delta=1.02 \mathrm{ppm}(\mathrm{s}, 6 \mathrm{H}$, $\left.\mathrm{CH}_{3}\right) ; 1.05 \mathrm{ppm}\left(\mathrm{s}, 9 \mathrm{H}, \mathrm{CH}_{3}\right) ; 2.26 \mathrm{ppm}\left(\mathrm{s}, 3 \mathrm{H}, \mathrm{CH}_{3}\right)$; $2.27\left(\mathrm{~s}, 2 \mathrm{H}, \mathrm{CH}_{3}\right) ; 6.51 \mathrm{ppm}(\mathrm{d}, J=8.0 \mathrm{~Hz}, 1 \mathrm{H}, \mathrm{Ar}-$ $\mathrm{H}) ; 6.61 \mathrm{ppm}(\mathrm{d}, J=8.0 \mathrm{~Hz}, 1 \mathrm{H}, \mathrm{Ar}-\mathrm{H}) ; 6.77-6.64$ ppm (m, 2H, Ar-H); $7.02 \mathrm{ppm}(\mathrm{d}, J=8.7 \mathrm{~Hz}, 4 \mathrm{H}, \mathrm{Ar}-$ $\mathrm{H}) ; 7.12-7.05 \mathrm{ppm}(\mathrm{m}, 5 \mathrm{H}, \mathrm{Ar}-\mathrm{H}) ; 7.15 \mathrm{ppm}(\mathrm{m}, 3 \mathrm{H}$, Ar-H); 7.21 ppm (m, 5H, Ar-H).

${ }^{13} \mathrm{C}$ NMR $\left(62.5 \mathrm{MHz}, \mathrm{CDCl}_{3}\right): \delta=21.41 ; 27.5 ; 27.6$; $37.27 ; 37.30 ; 120.72 ; 120.75 ; 121.80 ; 121.84 ; 125.6$; $126.0 ; 126.7 ; 127.0 ; 127.4 ; 127.7 ; 128.4 ; 129.0$; $129.7 ; 129.8 ; 129.9 ; 130.0 ; 130.7 ; 130.9 ; 136.4$; $137.1 ; 137.2 ; 137.6 ; 140.3 ; 140.9 ; 141.4 ; 167.22$; 167.24.

MS (EI, $70 \mathrm{eV}): \mathrm{m} / \mathrm{z}(\%): 367(100 \%) ; 310(63 \%)$; 296 (28\%); 282 (24\%); 267 (25\%); 178 (23\%); 165 (50\%); 57 (30\%).

\section{(E/Z)-4-((3-methylphenyl)(phenyl)methylene)-2-} tert-butyl-4H-benzo[d][1,3] oxazine ${ }^{10}(5 \mathrm{c})$

Prepared according to the general procedure from N(2-((3-methylphenyl)ethynyl)phenyl)pivalamide (102.0 mg, $0.35 \mathrm{mmol}$ ) with mesityl(phenyl)iodonium trifluoromethanesulfonate $(198.4 \mathrm{mg} 0.42 \mathrm{mmol})$. Reaction time: $12 \mathrm{~h}$. The crude product was purified by column chromatography on Brockmann II type neutral alumina with hexanes-dichloromethane 100:1 to give a yellow solid ( $85 \mathrm{mg}, 0.23 \mathrm{mmol}, 66 \%$ ). Isomer ratio $\mathrm{E} / \mathrm{Z}=2 / 3$ (from $1 \mathrm{H} \mathrm{NMR}$ ). 
Prepared according to the general procedure from N(2-(phenylethynyl)phenyl)pivalamide (97.1 $\mathrm{mg}, 0.35$ mmol) with (3-methylphenyl)(mesityl)iodonium trifluoromethanesulfonate $(204.2 \mathrm{mg} 0.42 \mathrm{mmol})$ Reaction time: $12 \mathrm{~h}$. The crude product was purified by column chromatography on Brockmann II type neutral alumina with hexanes-dichloromethane 100:1 to give a yellow solid (79 $\mathrm{mg}, 0.21 \mathrm{mmol}, 61 \%)$. Isomer ratio $\mathrm{E} / \mathrm{Z}=1.8 / 1$ (from ${ }^{\mathrm{H}} \mathrm{HMR}$ ).

Mp $128-130{ }^{\circ} \mathrm{C} . R_{f}: 0.67$ (hexanes-ethyl acetate, $7: 1)$;

IR (solid): 3055; 3017; 2972; 2928; 2865; 1639; 1597; $1455 ; 1219 ; 1139 ; 1062 \mathrm{~cm}^{-1}$.

${ }^{1} \mathrm{H}$ NMR $\left(250 \mathrm{MHz}, \mathrm{CDCl}_{3}\right): \delta=1.02 \mathrm{ppm}(\mathrm{s}, 4.5 \mathrm{H}$, $\left.\mathrm{CH}_{3}\right) ; 1.03 \mathrm{ppm}\left(\mathrm{s}, 9 \mathrm{H}, \mathrm{CH}_{3}\right) ; 2.18 \mathrm{ppm}(\mathrm{s}, 1.5 \mathrm{H}$, $\left.\mathrm{CH}_{3}\right) ; 2.22 \mathrm{ppm}\left(\mathrm{s}, 3 \mathrm{H}, \mathrm{CH}_{3}\right) ; 6.53 \mathrm{ppm}(\mathrm{t}, J=9.1 \mathrm{~Hz}$, $1.5 \mathrm{H}, \mathrm{Ar}-\mathrm{H}) ; 6.65-6.71 \mathrm{ppm}(\mathrm{m}, 1.5 \mathrm{H}, \mathrm{Ar}-\mathrm{H}) ; 6.92-$ $7.03 \mathrm{ppm}(\mathrm{m}, 4 \mathrm{H}, \mathrm{Ar}-\mathrm{H}), 7.06-7.09 \mathrm{ppm}(\mathrm{m}, 5.5 \mathrm{H}$, Ar-H); 7.13 - 7.24 ppm (m, 8H, Ar-H).

${ }^{13} \mathrm{C}$ NMR $\left(62.5 \mathrm{~Hz}, \mathrm{CDCl}_{3}\right): \delta=21.5 ; 21.6 ; 27.5$; $37.3 ; 120.9 ; 121.7 ; 125.56 ; 125.59 ; 126.0 ; 126.7$; $127.0 ; 127.1 ; 127.5 ; 127.6 ; 127.7 ; 127.75 ; 127.82$; $128.3 ; 128.9 ; 129.1 ; 129.8 ; 130.0 ; 130.8 ; 130.9$; $131.4 ; 137.0 ; 138.7 ; 139.9 ; 140.7 ; 141.3 ; 141.5$; 167.2; 167.3.

MS (EI, $70 \mathrm{eV}): \mathrm{m} / \mathrm{z}(\%): 367(100 \%) ; 310(60 \%)$; 296 (30\%); 282 (24\%); 267 (28\%); 178 (25\%); 165 (53\%); 57 (31\%).

\section{(E,Z)-2-tert-butyl-4-((2-}

methylphenyl)(phenyl)methylene)-4Hbenzo[d] [1,3] oxazine ${ }^{10}(5 d)$

Prepared according to the general procedure from N(2-((2-methylphenyl)ethynyl)phenyl)pivalamide

(102.0 mg, $0.35 \mathrm{mmol}$ ) with mesityl(phenyl)iodonium trifluoromethanesulfonate $(198.4 \mathrm{mg}, 0.42 \mathrm{mmol})$ Reaction time: $12 \mathrm{~h}$. The crude product was purified by column chromatography on silica gel hexanesethyl acetate, 50:1 to give a yellow oil $(91 \mathrm{mg}, 0.25$ mmol, $71 \%$ ). Isomer ratio $\mathrm{E} / \mathrm{Z}=1 / 1.2$ (from ${ }^{1} \mathrm{H}$ NMR).

$R_{f}: 0.73$ (hexanes-ethyl acetate, 7:1).

IR (thin film): 3058; 3018; 2970; 2928; 2867; 1642; $1598 ; 1456 ; 1273 ; 1218 ; 1139 ; 1061 ; 1029 \mathrm{~cm}^{-1}$.

${ }^{1} \mathrm{H}$ NMR $\left(250 \mathrm{MHz}, \mathrm{CDCl}_{3}\right): \delta=0.89 \mathrm{ppm}(\mathrm{s}, 7.5 \mathrm{H}$, $\left.\mathrm{CH}_{3}\right) ; 1.06 \mathrm{ppm}\left(\mathrm{s}, 9 \mathrm{H}, \mathrm{CH}_{3}\right) ; 2.01 \mathrm{ppm}\left(\mathrm{s}, 3 \mathrm{H}, \mathrm{CH}_{3}\right)$; $2.23 \mathrm{ppm}\left(\mathrm{s}, 2.5 \mathrm{H}, \mathrm{CH}_{3}\right) ; 6.32 \mathrm{ppm}(\mathrm{d}, J=7.7 \mathrm{~Hz}, 1 \mathrm{H}$, Ar-H); $6.66 \mathrm{ppm}$ (ddd, $J=8.6 \mathrm{~Hz}$ and $6.4 \mathrm{~Hz}$ and 2.3 $\mathrm{Hz}, 1 \mathrm{H}, \mathrm{Ar}-\mathrm{H}) ; 6.75$ ppm (m, 2H, Ar-H); 7.03 - 7.12 ppm (m, 12H, Ar-H); 7.15 - 7.27 ppm (m, 9H, Ar-H).

${ }^{13} \mathrm{C}$ NMR $\left(62.5 \mathrm{~Hz}, \mathrm{CDCl}_{3}\right): \delta=19.9 ; 20.1 ; 27.2$; $27.5 ; 37.2 ; 37.4 ; 119.4 ; 119.9 ; 121.1 ; 122.0 ; 125.7$; $125.8 ; 126.0 ; 126.1 ; 126.4 ; 126.6 ; 126.8 ; 127.1$; $127.3 ; 127.7 ; 128.0 ; 128.9 ; 129.7 ; 129.8 ; 130.0$; $130.1 ; 131.0 ; 131.2 ; 136.6 ; 137.8 ; 139.0 ; 139.7$; $139.9 ; 141.0 ; 141.4 ; 141.48 ; 141.8 ; 167.1 ; 167.4$.
MS (EI, $70 \mathrm{eV}): \mathrm{m} / \mathrm{z}(\%): 367$ (100\%); 310 (55\%); 296 (23\%); 282 (23\%); 178 (47\%); 165 (23\%); 57 $(55 \%)$.

\section{(E/Z)-2-tert-butyl-4-((4- methoxyphenyl)(phenyl)methylene)-4H- benzo[d][1,3]oxazine ${ }^{10}(5 e)$}

Prepared according to the general procedure from N(2-((4-methoxyphenyl)ethynyl)phenyl)pivalamide

(107.6 mg, $0.35 \mathrm{mmol}$ ) with mesityl(phenyl)iodonium trifluoromethanesulfonate $(198.4 \mathrm{mg} 0.42 \mathrm{mmol})$ Reaction time: $24 \mathrm{~h}$. The crude product was purified by column chromatography on silica gel with hexaneethyl acetate $50: 1$ to give a bright yellow oil $(50 \mathrm{mg}$, $0.13 \mathrm{mmol}, 37 \%$ ). Isomer ratio $\mathrm{E} / \mathrm{Z}=1 / 2$ (from $1 \mathrm{H}$ NMR)

$R_{f:} 0.61$ (hexanes-ethyl acetate, 7:1).

IR (thin film): 3058; 3031; 2958; 2925; 2853; 1639; $1601 ; 1509 ; 1456 ; 1245 ; 1218 ; 1171 ; 1137 ; 1032 ; 834$ $\mathrm{cm}^{-1}$.

${ }^{1} \mathrm{H}$ NMR $\left(250 \mathrm{MHz}, \mathrm{CDCl}_{3}\right): \delta=1.02 \mathrm{ppm}(\mathrm{s}, 4.5 \mathrm{H}$, $\left.\mathrm{CH}_{3}\right) ; 1.07 \mathrm{ppm}\left(\mathrm{s}, 9 \mathrm{H}, \mathrm{CH}_{3}\right) ; 3.73 \mathrm{ppm}\left(\mathrm{s}, 3 \mathrm{H}, \mathrm{CH}_{3}\right)$; $3.74 \mathrm{ppm}\left(\mathrm{s}, 1.5 \mathrm{H}, \mathrm{CH}_{3}\right) ; 6.50 \mathrm{ppm}(\mathrm{d}, \mathrm{J}=8.0 \mathrm{~Hz}, 1 \mathrm{H}$, Ar-H); $6.72-6.59(\mathrm{~m}, 1.5 \mathrm{H}, \mathrm{Ar}-\mathrm{H}) ; 6.77 \mathrm{ppm}(\mathrm{d}, J=$ $8.8 \mathrm{~Hz}, 3 \mathrm{H}, \mathrm{Ar}-\mathrm{H}) ; 7.11-7.05 \mathrm{ppm}(\mathrm{m}, 4 \mathrm{H}, \mathrm{Ar}-\mathrm{H})$; $7.19-7.12 \mathrm{ppm}(\mathrm{m}, 5 \mathrm{H}, \mathrm{Ar}-\mathrm{H}) ; 7.24-7.19 \mathrm{ppm}(\mathrm{m}$, $5 \mathrm{H})$.

${ }^{13} \mathrm{C}$ NMR $\left(62.5 \mathrm{~Hz}, \mathrm{CDCl}_{3}\right): \delta=27.5 ; 27.6 ; 37.27$; 37.33; 55.4; 113.1; 114.5; 120.5; 121.9; 122.0; 125.55; $125.61 ; 125.95 ; 126.03 ; 127.0 ; 127.5 ; 127.7 ; 129.0$; $129.7 ; 129.8 ; 130.0 ; 131.0 ; 131.3 ; 132.0 ; 132.6$; $140.9 ; 141.1 ; 141.3 ; 158.4 ; 167.3$.

\section{(E,Z)-2-tert-butyl-4-((4- chlorophenyl)(phenyl)methylene)-4H- benzo[d][1,3] oxazine ${ }^{10}(5 f)$}

Prepared according to the general procedure from N(2-((4-chlorophenyl)ethynyl)phenyl)pivalamide

(109.1 mg, $0.35 \mathrm{mmol}$ ) with mesityl(phenyl)iodonium trifluoromethanesulfonate $(198.4 \mathrm{mg} 0.42 \mathrm{mmol})$ Reaction time: $16 \mathrm{~h}$. The crude product was purified by column chromatography on Brockmann II type neutral alumina with hexane-dichloromethane 100:1 to give a pale yellow solid (92 $\mathrm{mg}, 0.24 \mathrm{mmol}, 68 \%$ ). Isomer ratio $\mathrm{E} / \mathrm{Z}=1 / 2.3$ (from ${ }^{1} \mathrm{H} \mathrm{NMR}$ ).

Prepared according to the general procedure from N(2-(phenylethynyl)phenyl)pivalamide (97.1 mg, 0.35 mmol) with (4-chlorophenyl)(mesityl)iodonium trifluoromethanesulfonate $(212.8 \mathrm{mg} \quad 0.42 \mathrm{mmol})$ Reaction time: $16 \mathrm{~h}$. The crude product was purified by column chromatography on Brockmann II type neutral alumina with hexane/ dichloromethane 100/ 1 to give a pale yellow solid $(73 \mathrm{mg}, 0.19 \mathrm{mmol}, 54 \%)$. Isomer ratio $\mathrm{E} / \mathrm{Z}=9 / 5$ (from $1 \mathrm{H}$ NMR)

Mp $121-123{ }^{\circ} \mathrm{C} . R f: 0.70$ (hexanes-ethyl acetate, $7: 1)$;

IR (solid): 2695; 1638; 1594; 1487; 1455; 1219; 1140; $1087 ; 1059 ; 1015 ; 832 ; 757 \mathrm{~cm}^{-1}$. 
${ }^{1} \mathrm{H}$ NMR $\left(250 \mathrm{MHz}, \mathrm{CDCl}_{3}\right): \delta=1.01 \mathrm{ppm}(\mathrm{s}, 3.5 \mathrm{H}$, $\left.\mathrm{CH}_{3}\right) ; 1.05 \mathrm{ppm}\left(\mathrm{s}, 9 \mathrm{H}, \mathrm{CH}_{3}\right) ; 6.51 \mathrm{ppm}(\mathrm{d}, J=7.8$ $\mathrm{Hz}, 1 \mathrm{H}, \mathrm{Ar}-\mathrm{H}) ; 6.60 \mathrm{ppm}(\mathrm{d}, J=7.9 \mathrm{~Hz}, 1 \mathrm{H}, \mathrm{Ar}-\mathrm{H})$; $6.69 \mathrm{ppm}(\mathrm{m}, 1 \mathrm{H}, \mathrm{Ar}-\mathrm{H}) ; 6.76 \mathrm{ppm}(\mathrm{m}, 0.4 \mathrm{H}, \mathrm{Ar}-\mathrm{H})$; $7.04-7.14$ ppm (m, 6.5H, Ar-H); $7.16-7.24$ ppm (m, $10 \mathrm{H}, \mathrm{Ar}-\mathrm{H})$.

${ }^{13} \mathrm{C}$ NMR $\left(62.5 \mathrm{~Hz}, \mathrm{CDCl}_{3}\right): \delta=27.5 ; 27.6 ; 37.3$; $119.3 ; 119.5 ; 121.2 ; 121.3 ; 125.76 ; 125.82 ; 126.1$; $126.2 ; 126.9 ; 127.0 ; 127.7 ; 127.9 ; 128.0 ; 129.2$; $129.3 ; 130.0 ; 130.1 ; 130.2 ; 130.9 ; 131.4 ; 132.3$; $133.4 ; 138.6 ; 139.2 ; 139.7 ; 140.3 ; 141.3 ; 141.4$; $142.1 ; 142.2 ; 166.8 ; 167.1$.

MS (EI, $70 \mathrm{eV}): \mathrm{m} / \mathrm{z}(\%): 387$ (100\%); $330(50 \%)$; 295 (20\%); 267 (34\%); 239 (20\%); 165 (65\%); 57 (35\%).

\section{2-tert-butyl-4-((4-nitrophenyl)(phenyl)methylene)- 4H-benzo[d] $[1,3]$ oxazine $^{10}(5 \mathrm{~g})$}

Prepared according to the general procedure from N(2-((4-nitrophenyl)ethynyl)phenyl)pivalamide (112.8 $\mathrm{mg}, \quad 0.35 \mathrm{mmol})$ with mesityl(phenyl)iodonium trifluoromethanesulfonate $(198.4 \mathrm{mg} 0.42 \mathrm{mmol})$ Reaction time: $17 \mathrm{~h}$. The crude product was purified by column chromatography on Brockmann II type neutral alumina with hexanes-dichloromethane 100:1 to give a bright yellow solid $(70 \mathrm{mg}, 0.18 \mathrm{mmol}$, $50 \%$ ). Isomer ratio $\mathrm{E} / \mathrm{Z}=2 / 7$ (from $1 \mathrm{H} \mathrm{NMR}$ ).

Mp 130-132 ${ }^{\circ}$ C. $R_{f}: 0.53$ (hexanes-ethyl acetate 7:1); IR (solid): 3061; 2958; 2922; 2851; 1639; 1578; 1511; $1455 ; 1337 ; 1272 ; 1218 ; 1139 ; 1061 \mathrm{~cm}^{-1}$.

${ }^{1} \mathrm{H}$ NMR $\left(250 \mathrm{MHz}, \mathrm{CDCl}_{3}\right): \delta=1.05 \mathrm{ppm}(\mathrm{s}, 2 \mathrm{H}$, $\left.\mathrm{CH}_{3}\right) ; 1.09 \mathrm{ppm}\left(\mathrm{s}, 7 \mathrm{H}, \mathrm{CH}_{3}\right) ; 6.57(\mathrm{~d}, J=8.0 \mathrm{~Hz}, 1 \mathrm{H}$, $\mathrm{Ar}-\mathrm{H}) ; 6.77 \mathrm{ppm}(\mathrm{t}, J=6.2 \mathrm{~Hz}, 1 \mathrm{H}, \mathrm{Ar}-\mathrm{H}) ; 7.23-7.11$ ppm (m, 4H, Ar-H); 7.34 - 7.26 ppm (m, 3H, Ar-H); $7.42 \mathrm{ppm}(\mathrm{d}, J=8.6 \mathrm{~Hz}, 2 \mathrm{H} \mathrm{Ar}-\mathrm{H}) ; 8.11 \mathrm{ppm}(\mathrm{d}, J=$ $8.4 \mathrm{~Hz}, 2 \mathrm{H}, \mathrm{Ar}-\mathrm{H})$.

${ }^{13} \mathrm{C}$ NMR $\left(62.5 \mathrm{~Hz}, \mathrm{CDCl}_{3}\right): \delta=27.5 ; 27.6 ; 37.4$; $118.2 ; 120.6 ; 123.2 ; 124.2 ; 126.1 ; 126.2 ; 126.4$; $126.9 ; 127.3 ; 127.5 ; 128.20 ; 128.22 ; 129.0 ; 129.6$; $130.2 ; 130.8 ; 130.9 ; 131.1 ; 131.9 ; 139.7 ; 141.4$; $144.1 ; 146.2 ; 147.2 ; 166.2$.

MS (EI, $70 \mathrm{eV}): \mathrm{m} / \mathrm{z}(\%): 398(100 \%) ; 341(38 \%)$; 295 (70\%); 267 (50\%); 239 (33\%); 165 (40\%); 57 $(65 \%)$.

\section{4-(bis(4-methylphenyl)methylene)-2-tert-butyl-4H- benzo[d][1,3]oxazine ${ }^{10}(5 \mathrm{i})$}

Prepared according to the general procedure from N(2-((4-methylphenyl)ethynyl)phenyl)pivalamide $(102.0 \quad \mathrm{mg}, \quad 0.35 \mathrm{mmol})$ with 4methylphenyl(mesityl)iodonium trifluoromethanesulfonate (204.2 mg $0.42 \mathrm{mmol}$ ) Reaction time: $12 \mathrm{~h}$. The crude product was purified by column chromatography on Brockmann II type neutral alumina with hexanes-dichloromethane $50: 1$ to give a pale yellow solid (42 mg, $0.11 \mathrm{mmol}, 31 \%$ ).

Mp 138-140 ${ }^{\circ}$ C. $R_{f}: 0.72$ (hexanes-ethyl acetate, $7: 1$ );

IR (solid): 2958; 2921; 1638; 1602; 1509; 1455; 1273; $1219 ; 1137 ; 1059 ; 1021 ; 818 \mathrm{~cm}^{-1}$.

${ }^{1} \mathrm{H}$ NMR $\left(250 \mathrm{MHz}, \mathrm{CDCl}_{3}\right): \delta=1.04 \mathrm{ppm}(\mathrm{s}, 9 \mathrm{H}$, $\left.\mathrm{CH}_{3}\right) ; 2.26 \mathrm{ppm}\left(\mathrm{s}, 3 \mathrm{H}, \mathrm{CH}_{3}\right) ; 2.27 \mathrm{ppm}\left(\mathrm{s}, 3 \mathrm{H}, \mathrm{CH}_{3}\right)$; $6.57 \mathrm{ppm}(\mathrm{d}, J=7.9 \mathrm{~Hz} ; 1 \mathrm{H}$, Ar-H); $6.71 \mathrm{ppm}(\mathrm{dt}, J=$ $8.6 \mathrm{~Hz}$ and $4.4 \mathrm{~Hz}, 1 \mathrm{H}, \mathrm{Ar}-\mathrm{H}) ; 7.00 \mathrm{ppm}(\mathrm{s}, 1 \mathrm{H}, \mathrm{Ar}-$ $\mathrm{H}) ; 7.03$ - $7.15 \mathrm{ppm}$ (m, 10H, Ar-H).

${ }^{13} \mathrm{C} \mathrm{NMR}\left(62.5 \mathrm{~Hz}, \mathrm{CDCl}_{3}\right): \delta=21.43 ; 21.45 ; 27.5$; $37.28 ; 120.7 ; 122.0 ; 125.5 ; 125.9 ; 127.0 ; 128.4$; $129.6 ; 129.8 ; 129.9 ; 130.8 ; 136.4 ; 137.2 ; 137.3$; $137.8 ; 141.1 ; 141.3 ; 167.3$.

MS (EI, $70 \mathrm{eV}): \mathrm{m} / \mathrm{z}(\%): 381(100 \%) ; 324(50 \%)$; 310 (32\%); 296 (15\%); 281 (18\%); 179 (33\%); 57 $(35 \%)$.

\section{4-(bis(3-methylphenyl)methylene)-2-tert-butyl-4H- benzo[d][1,3] oxazine ${ }^{10}(5 j)$}

Prepared according to the general procedure from $\mathrm{N}$ (2-((3-methylphenyl)ethynyl)phenyl)pivalamide

$(102.0 \mathrm{mg}, \quad 0.35 \mathrm{mmol})$ with (3methylphenyl)(mesityl)iodonium

trifluoromethanesulfonate $(204.2 \mathrm{mg} 0.42 \mathrm{mmol})$ Reaction time: $12 \mathrm{~h}$. The crude product was purified by column chromatography on Brockmann II type neutral alumina with hexanes-dichloromethane 100:1 to give a yellow solid (81 $\mathrm{mg}, 0.21 \mathrm{mmol}, 61 \%)$.

Mp 103-105 ${ }^{\circ}$ C. $R_{f}: 0.79$ (hexanes-ethyl acetate, 7:1); IR (solid): 3033, 2958, 2924, 1642, 1598, 1456, 1218, $1137,1063 \mathrm{~cm}^{-1}$.

${ }^{1} \mathrm{H}$ NMR $\left(250 \mathrm{MHz}, \mathrm{CDCl}_{3}\right): \delta=1.03 \mathrm{ppm}(\mathrm{s}, 9 \mathrm{H}$, $\left.\mathrm{CH}_{3}\right) ; 2.18 \mathrm{ppm}\left(\mathrm{s}, 3 \mathrm{H}, \mathrm{CH}_{3}\right) ; 2.23 \mathrm{ppm}\left(\mathrm{s}, 3 \mathrm{H}, \mathrm{CH}_{3}\right)$; $6.53 \mathrm{ppm}(\mathrm{d}, J=8.1 \mathrm{~Hz}, 1 \mathrm{H}, \mathrm{Ar}-\mathrm{H}) ; 6.69 \mathrm{ppm}(\mathrm{ddd}, J$ $=8.4 \mathrm{~Hz}$ and $5.3 \mathrm{~Hz}$ and $3.4 \mathrm{~Hz}, 1 \mathrm{H}, \mathrm{Ar}-\mathrm{H}) ; 6.92-$ $7.01 \mathrm{ppm}(\mathrm{m}, 5 \mathrm{H}, \mathrm{Ar}-\mathrm{H}) ; 7.05-7.13 \mathrm{ppm}(\mathrm{m}, 5 \mathrm{H}, \mathrm{Ar}-$ $\mathrm{H})$.

${ }^{13} \mathrm{C}$ NMR $\left(62.5 \mathrm{~Hz}, \mathrm{CDCl}_{3}\right): \delta=21.5 ; 21.6 ; 27.5$; $37.3 ; 120.9 ; 121.8 ; 125.6 ; 126.0 ; 127.00 ; 127.03$; $127.5 ; 127.6 ; 127.8 ; 128.3 ; 128.9 ; 129.8 ; 130.7$; $131.4 ; 137.0 ; 138.7 ; 140.0 ; 140.5 ; 141.3 ; 141.4$; 167.2.

MS (EI, $70 \mathrm{eV}): \mathrm{m} / \mathrm{z}(\%): 381(100 \%) ; 324(48 \%)$; 310 (37\%); 281 (15\%); 178 (24\%); 57 (28\%).

\section{4-(bis(4-chlorophenyl)methylene)-2-tert-butyl-4H- benzo[d] $[1,3]$ oxazine $^{10}(5 \mathrm{k})$}

Prepared according to the general procedure from $\mathrm{N}$ (2-((4-chlorophenyl)ethynyl)phenyl)pivalamide

$(109.1 \mathrm{mg}, \quad 0.35 \mathrm{mmol})$ with (4chlorophenyl)(mesityl)iodonium trifluoromethane sulfonate (212.8 mg $0.42 \mathrm{mmol}$ ) Reaction time: $24 \mathrm{~h}$. The crude product was purified by column chromatography on Brockmann II type neutral alumina with hexanes-dichloromethane 100:1 to give a pale yellow solid (87 mg, $0.21 \mathrm{mmol}, 59 \%)$.

Mp $120-122{ }^{\circ} \mathrm{C} . R_{f}:=0.79$ (hexanes-ethyl acetate, $7: 1)$;

IR (solid): 2963; 1636; 1608; 1585; 1489; 1455; 1217; $1140 ; 1088 ; 1012 ; 827 \mathrm{~cm}^{-1}$. 
${ }^{1} \mathrm{H}$ NMR $\left(250 \mathrm{MHz}, \mathrm{CDCl}_{3}\right): \delta=1.13 \mathrm{ppm}(\mathrm{s}, 9 \mathrm{H}$, $\left.\mathrm{CH}_{3}\right) ; 6.67 \mathrm{ppm}(\mathrm{d}, J=7.9 \mathrm{~Hz}, 1 \mathrm{H}, \mathrm{Ar}-\mathrm{H}) ; 6.87 \mathrm{ppm}$ (t, $J=7.0 \mathrm{~Hz}, 1 \mathrm{H}, \mathrm{Ar}-\mathrm{H}) ; 7.16 \mathrm{ppm}(\mathrm{d}, J=8.5 \mathrm{~Hz}$, 2H, Ar-H); $7.22 \mathrm{ppm}(\mathrm{d}, J=6.4 \mathrm{~Hz}, 4 \mathrm{H}, \mathrm{Ar}-\mathrm{H}) ; 7.35-$ $7.26 \mathrm{ppm}(\mathrm{m}, 4 \mathrm{H}, \mathrm{Ar}-\mathrm{H})$.

${ }^{13} \mathrm{C}$ NMR $\left(62.5 \mathrm{~Hz}, \mathrm{CDCl}_{3}\right): \delta=27.6 ; 37.3 ; 118.1$; $121.0 ; 126.0 ; 126.3 ; 126.9 ; 128.1 ; 129.5 ; 130.4$; $131.4 ; 132.4 ; 132.7 ; 133.7 ; 138.2 ; 138.9 ; 141.4$; $142.6 ; 166.8$.

MS (EI, $70 \mathrm{eV}): \mathrm{m} / \mathrm{z}(\%): 421$ (91\%); $364(42 \%) ; 329$ (52\%); 301 (35\%); 267 (45\%); 239 (45\%); 199 (90\%); 190 (22\%); 163 (57\%); 57 (100\%).

\section{4-(diphenylmethylene)-2-phenyl-4H- benzo[d][1,3] oxazine ${ }^{10}(5 \mathrm{l})$}

$\mathrm{N}$-(2-(phenylethynyl)phenyl)benzamide (104.1 mg, 0.35 mmol, 1.0 equiv.), aryl(mesityl)iodonium trifluoromethane sulfonate $(198.3 \mathrm{mg}, 0.42 \mathrm{mmol}, 1.2$ equiv.) and $\mathrm{Cu} / \mathrm{Fe}(22.2 \mathrm{mg}, 0.0175 \mathrm{mmol}, 5 \mathrm{~mol} \%)$ were placed in a $4 \mathrm{ml}$ vial, which was sealed with rubber septa, evacuated then charged with argon. 1,2dichloroethane $(3 \mathrm{ml})$ was added, and the reaction mixture was stirred at $50^{\circ} \mathrm{C}$ for 17 hours. The catalyst was separated from the reaction mixture with a magnet. The reaction mixture was diluted with $\mathrm{CH}_{2} \mathrm{Cl}_{2}(15 \mathrm{ml})$, washed twice with saturated sodium bicarbonate solution $(15 \mathrm{ml})$, the aqueous phase was extracted with $\mathrm{CH}_{2} \mathrm{Cl}_{2}(15 \mathrm{ml})$, and the combined organic phases were dried over magnesium sulfate and the solvent was evaporated in vacuo. The crude product was purified by column chromatography on silica gel with hexane-ethyl acetate 50:1 to give a bright yellow solid (91 mg, $0.24 \mathrm{mmol}, 70 \%)$.

Mp $187-189{ }^{\circ} \mathrm{C} . R_{f}=0.64$ (hexanes-ethyl acetate, $7: 1)$;

IR (solid): 3055; 1738; 1633; 1612; 1591; 1574; 1449; $1243 ; 1069 ; 1024 \mathrm{~cm}^{-1}$.

${ }^{1} \mathrm{H}$ NMR $\left(250 \mathrm{MHz}, \mathrm{CDCl}_{3}\right): \delta=6.58 \mathrm{ppm}(\mathrm{d}, \mathrm{J}=7.8$ $\mathrm{Hz}, 1 \mathrm{H}, \mathrm{Ar}-\mathrm{H}) ; 6.72 \mathrm{ppm}(\mathrm{t}, \mathrm{J}=7.6 \mathrm{~Hz}, 1 \mathrm{H}, \mathrm{Ar}-\mathrm{H})$; $7.11 \mathrm{ppm}(\mathrm{t}, \mathrm{J}=7.5 \mathrm{~Hz}, 1 \mathrm{H}$, Ar-H); $7.35-7.19 \mathrm{ppm}$ (m, 14H, Ar-H); 7.79 ppm (d, J= 7.4 Hz, 2H, Ar-H).

${ }^{13} \mathrm{C}$ NMR $\left(62.5 \mathrm{MHz}, \mathrm{CDCl}_{3}\right): \delta=121.5 ; 122.0$; $126.0 ; 126.4 ; 127.1 ; 127.2 ; 127.7 ; 128.0 ; 128.1$; $128.4 ; 129.2 ; 130.1 ; 130.9 ; 131.2 ; 131.7 ; 140.1$; $140.6 ; 141.5 ; 141.6 ; 156.2$.

MS (EI, $70 \mathrm{eV}): \mathrm{m} / \mathrm{z}(\%): 373$ (97\%); $356(10 \%) ; 344$ $(66 \%) ; 267(20 \%) ; 239(42 \%) ; 179(30 \%) ; 165$ (100\%); 77 (20\%).

\section{Acknowledgment}

This project was supported by the "Lendület" Research Scholarship of the Hungarian Academy of Sciences, and by OTKA-NKTH (CK 80763). The authors also thank Dr. Szabolcs Béni (Semmelweis University, Budapest) for the HRMS measurements and Prof. Tim Peelen (Lebanon Valley College) for the proofreading of this manuscript.

\section{References}

(1) (a) Hassan, J.; Sévignon, M.; Gozzi, C.; Schulz, E.; Lemaire, M.; Chem. Rev., 2002, 102, 1359-1470. (b) Alberico, D.; Scott, M. E.; Lautens, M. Chem. Rev., 2007, 107, 174-238. (c) Stuart, D. R.; Fagnou, K. Science, 2007, 316, 1172-1175. (d) Ackermann, L.; Vicente, R.; Kapdi, A. R. Angew. Chem., Int. Ed., 2009, 48, 9792-9826. (e) Bellina, F.; Rossi, R. Tetrahedron, 2009, 65, 10269-10310. (f) Joucla, L.; Djakovitch, L. Adv. Synth. Catal., 2009, 351, 673-714. (g) Chen, X.; Engle, K. M.; Wang, D.-H.; Yu, J.-Q. Angew. Chem., Int. Ed., 2009, 48, 5094-5115. (h) Satoh, T.; Miura, M. Chemistry Letters 2007, 36, 200-205. (i) Stuart, D. R.; Villemure, E.; Fagnou, K. J. Am. Chem. Soc., 2007, 129, 12072-12073. (j) Campeau, L.-C.; Schipper, D. J.;

Fagnou, K. J. Am. Chem. Soc., 2008, 130, 3266-3267. (k) Godula, K.; Sames, D. Science, 2006, 312, 67-72. (1) Crabtree, R. H. J. Chem. Soc., Dalton Trans., 2001, 2437-2450. (n) Wencel-Delord, J.; Droge, T.; Liu, F.; Glorius, F. Chem. Soc. Rev., 2011, 40, 4740-4761. (o) Mei T.-S., Kou L.; Ma S.; Engle K. M.; Yu J.-Q. Synthesis, 2012, 44, 1778-1791. (p) Crabtree, R. H. Chem. Rev., 2010, 110, 575-575. (q) Lyons, T. W.; Sanford, M. S. Chem. Rev., 2010, 110, 1147-1169. (r) Neufeldt, S. R.; Sanford, M. S. Acc. Chem. Res., 2012, 45, 936-946.

(2) (a) Barder, T. E.; Walker, S. D.; Martinelli, J. R.; Buchwald, S. L. J. Am. Chem. Soc., 2005, 127, 46854696. (b) Molander, G. A.; Ellis, N. Acc. Chem. Res., 2007, 40, 275-286. (c) Zhang, H.; Cai, Q.; Ma, D. J. Org. Chem., 2005, 70, 5164-5173. (d) Ley, S. V.; Thomas, A. W. Angew. Chem., Int. Ed., 2003, 42, 5400 5449. (e) Mowery, M. E.; DeShong, P. J. Org. Chem., 1999, 64, 1684-1688. (f) Wentzel, M. T.; Hewgley, J. B.; Kamble, R. M.; Wall, P. D.; Kozlowski, M. C. Adv. Synth. Catal., 2009, 351, 931-937. (g) Britcher, L. G.; Kehoe, D. C.; Matisons, J. G.; Swincer, A. G. Macromolecules 1995, 28, 3110-3118.

(3) (a) Hypervalent Iodine Chemistry. In Topics in Current Chemistry; Wirth, T., Ed.; Springer: New York, 2003; 224, 1-265. (b) Marcin Bielawski: Diaryliodonium Salts, Stockholm, 2011. (c) Merritt, E. A.; Olofsson, B. Angew. Chem., Int. Ed., 2009, 48, 9052-9070. (d) Kalyani, D.; Deprez, N. R.; Desai, L. V.; Sanford, M. S. J. Am. Chem. Soc., 2005, 127, 7330-7331. (e) Parra, A.; Reboredo, S. Chem. - Eur. J., 2013, 19, 17244-17260. (f) Merritt E. A.; Malmgren J.; Klinke F. J.; Olofsson B. Synlett, 2009, 14, 2277-2280. (g) Bielawski, M.; Zhu, M.; Olofsson, B. Adv. Synth. Catal., 2007, 349, 26102618.

(4) (a) Daugulis, O.; Zaitsev, V. G. Angew. Chem., Int. Ed., 2005, 44, 4046-4048. (b) Yang, S.; Li, B.; Wan, X.; Shi, Z. J. Am. Chem. Soc., 2007, 129, 6066-6067. (c) Brasche, G.; García-Fortanet, J.; Buchwald, S. L. Org. Lett., 2008, 10, 2207-2210. (d) Wang, G.-W.; Yuan, T.T.; Wu, X.-L. J. Org. Chem., 2008, 73, 4717-4720. (e) Ciana, C.-L.; Phipps, R. J.; Brandt, J. R.; Meyer, F.-M.; Gaunt, M. J. Angew. Chem., Int. Ed., 2011, 50, 458-462.

(5) (a) Zhang, Y.-H.; Shi, B.-F.; Yu, J.-Q. J. Am. Chem. Soc., 2009, 131, 5072-5074. (b) Zhou, Y.; Zhao, J.; Liu, L. Angew. Chem., Int. Ed., 2009, 48, 7126-7128. (c) Bedford, R. B.; Mitchell, C. J.; Webster, R. L. Chem. Commun., 2010, 46, 3095-3097. (d) Cornella, J.; Righi, M.; Larrosa, I. Angew. Chem., Int. Ed., 2011, 50, 94299432. (e) Akai, S.; Peat, A. J.; Buchwald, S. L. J. Am. Chem. Soc., 1998, 120, 9119-9125. (f) Do, H.-Q.; Khan, R. M. K.; Daugulis, O. J. Am. Chem. Soc., 2008, 130, 
15185-15192. (g) Chen, B.; Hou, X.-L.; Li, Y.-X.; Wu, Y.-D. J. Am. Chem. Soc., 2011, 133, 7668-7671.

(6) (a) Phipps, R. J.; Grimster, N. P.; Gaunt, M. J. J. Am. Chem. Soc., 2008, 130, 8172-8174. (b) Deprez, N.

R.; Kalyani, D.; Krause, A.; Sanford, M. S. J. Am.

Chem. Soc., 2006, 128, 4972-4973.

(7) Phipps, R. J.; Gaunt, M. J. Science, 2009, 323, 15931597.

(8) Zhu, S.; MacMillan, D. W. C. J. Am. Chem. Soc., 2012, 134, 10815-10818.

(9) Cahard, E.; Bremeyer, N.; Gaunt, M. J. Angew. Cem. Int. Ed., 2013, 52, 9284-9288.

(10) Sinai, Á.; Mészáros, Á.; Gáti, T.; Kudar, V.; Palló, A.; Novák, Z. Org. Lett., 2013, 15, 5654-5657.

(11) (a) Tang, B.-X.; Song, R.-J.; Wu, C.-Y.; Liu, Y.; Zhou, M.-B.; Wei, W.-T.; Deng, G.-B.; Yin, D.-L.; Li, J.-H. J. Am. Chem. Soc., 2010, 132, 8900-8902. (b) Duong, H. A.; Gilligan, R. E.; Cooke, M. L.; Phipps, R. J.; Gaunt, M. J. Angew. Chem., 2011, 123, 483-486. (c) Daugulis, O.; Do, H.-Q.; Shabashov, D. Acc. Chem. Res., 2009, 42, 1074-1086. (d) Fujihara, T.; Xu, T.; Semba, K.; Terao, J.; Tsuji, Y. Angew. Chem., Int. Ed., 2011, 50, 523-527. (e) Phipps, R. J.; McMurray, L.; Ritter, S.; Duong, H. A.; Gaunt, M. J. J. Am. Chem. Soc., 2012, 134, 10773-10776. (f) Beletskaya, I. P.; Cheprakov, A. V. Coord. Chem. Rev., 2004, 248, 2337-2364.

(12) B.Chen, X. Hou, Y. Li, Y. Wu, J. Am. Chem. Soc. 2011, 133, 7668-7671.

(13) Lee, E. Y.; Park, J. ChemCatChem, 2011, 3, 1127-1129.

(14) Kovács, Sz.; Zih-Perényi K.; Révész, Á.; Novák Z. Synthesis, 2012, 44, 3722-3730.

(15) (a) Kovács, Sz.; Novak, Z. Org. Biomol. Chem., 2011, 9 , 711-716. (b) Kovács, Sz.; Novák, Z. Tetrahedron, 2013, 69, 8987-8993.

(16) Bedford, R. B.; Haddan, M. F.; Webster, R. L.; Mitchell, C. I. Angew. Chem., Int. Ed., 2011, 50, 5524-5527.

(17) Bielawski, M.; Zhu, M.; Olofsson, B. Adv. Synth. Catal., 2007, 349, 2610-2618.

(18) Brasche, G.; Garcia-Fortanet, J.; Buchwald, S. L. Org. Lett., 2008, 10, 2207-2210.

(19) Sanford, G. Sci. Synth., 2007, 31, 21-78. 
Please place the graphical abstract and short title of the article here. The short title will be used as a running header.

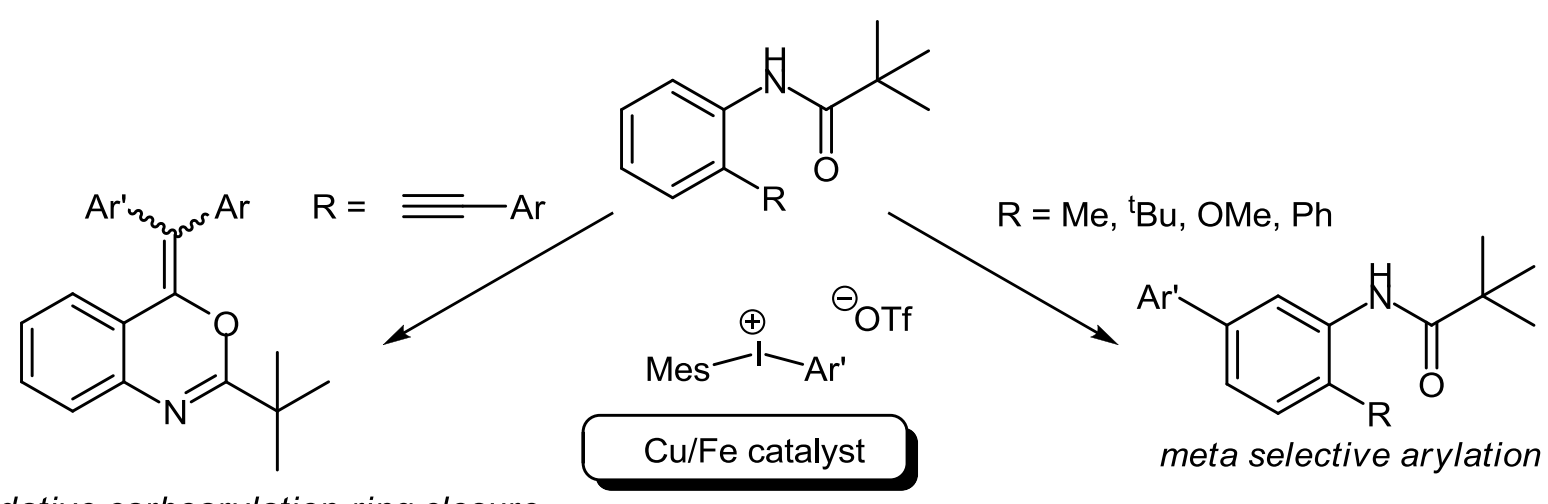

oxydative carboarylation-ring closure

Synthesis of benzoxazines

15 examples, $47-83 \%$

14 examples, $31-71 \%$

Short title: $\mathrm{Cu} / \mathrm{Fe}$ catalyzed oxidative arylations of anilides

\section{Manuscript submission checklist}

- Statement of significance of work.

- Full mailing address, telephone, and fax numbers and e-mail address of the corresponding author.

- Graphical abstract.

- 5 key words.

- Original Word file.

- Original graphics files.

Proceed to submit your article via our online submission system at http://mc.manuscriptcentral.com/synthesis. 